Document ID \#P111125

\title{
Water quality trends in the Entiat River subbasin: Final 2008 annual report to BPA and NOAA Fisheries
}

\author{
$3 / 16 / 2009$ \\ Richard Woodsmith and Andy Bookter \\ PNW Research Station, USDA Forest Service, Wenatchee, WA
}

Project Title: Integrated Status and Effectiveness Monitoring Program

Project \# 2003-017-00

Contract Title: 2003-017-00 EXP ISEMP / USFS-PNW LAB -ENTIAT MONITORING

Contract \# 37005

Work Element: F: 157. Collect/Generate/Validate Field and Lab Data - Measure, analyze and interpret water quality trends in Entiat River subbasin

Performance Period: 4/1/2008 - 3/31/2009

Submitted to: Chris Jordan, NFSC, Pamela Nelle, ISEMP Coordinator, David Byrnes, BPA Project Manager / COTR

Submitted by: Richard Woodsmith and Andy Bookter, PNW Research Station, USDA Forest

Service

\begin{abstract}
The ISEMP program monitors the status and trend of water quality elements that may affect restoration project effectiveness in the Entiat subbasin. As part of this effort, the PNW Research Station (PNW) measures, analyzes and interprets temporal trends in natural stream water $\mathrm{pH}$, dissolved oxygen, specific conductivity and temperature. The Entiat River is currently on the Clean Water Act 303(d) list for $\mathrm{pH}$ exceedence, and there is insufficient information to determine the spatial and temporal extent or potential causes of this exceedence. In the spring 2008, PNW redeployed data-logging, multiparameter probes at four locations in the Entiat subbasin to measure water quality parameters, focusing on $\mathrm{pH}$. This resumed previous data collection that was interrupted by river ice in early December 2007. Instruments were again removed from the river in early December 2008. This annual report covers the period from December 2007 through December 2008.

The highest $\mathrm{pH}$ values occurred during the low-flow period from midsummer through the following midspring then dropped sharply during the annual snowmelt runoff period from late spring through early summer. Water temperature began rapidly increasing during the receding limb of the annual snowmelt hydrograph. Highest mean monthly temperatures occurred in July and August, while instantaneous maxima occurred during the period July-September. Dissolved oxygen reached its lowest levels during the period of highest water temperature in JulySeptember. Specific conductivity remained very low at all sites throughout the year.
\end{abstract}




\section{Introduction}

Production of high-quality water is a vitally important ecosystem service in the largely semi-arid interior Columbia River basin (ICRB). Communities, tribes, and local, state, and federal agencies are concerned about maintenance of this water supply for domestic, agricultural, livestock, industrial, recreational, and ecosystem uses. A sufficient supply of high-quality water is also essential for an ongoing, multi-agency effort to restore depleted salmonid populations in the ICRB (UCSRB 2007). The Bonneville Power Administration (BPA) is a primary sponsor of this restoration effort (BPA 2008). BPA funds the National Oceanic and Atmospheric Administration (NOAA-Fisheries), Integrated Status and Effectiveness Monitoring Program (ISEMP). ISEMP supports the development of a region-wide research, monitoring, and evaluation program to assess the status and trends of anadromous salmonid populations, their tributary habitat, and effects of restoration and management actions in the ICRB (ISEMP 2008). One of ISEMP's tasks is to support long-term status and trend monitoring of water quality parameters that may compromise effectiveness of salmonid restoration projects. A particularly active program of salmonid habitat restoration is underway in the Entiat subbasin, where all salmonid populations have been in decline since European settlement (CCCD 2004). The subbasin currently provides critical habitat for three of these populations listed under the Endangered Species Act, including spring Chinook salmon (Oncorhynchus tshawytscha) (endangered), summer steelhead (Oncorhynchus mykiss) (endangered), and bull trout (Salvelinus confluentus) (threatened) (CCCD 2004, UCSRB 2007).

As part of a coordinated federal and state effort to monitor compliance with the federal Clean Water Act of 1977, point measurements of water quality are taken monthly at Entiat River mile 1.4 by the Washington State Department of Ecology (WDOE). In the 2008 WDOE Water Quality Assessment 305(b) report and 303(d) list, occasional exceedences of the state water quality standard for $\mathrm{pH}$ are documented for the period from 2004 to 2006 (WDOE 2009). As a result of these exceedences, the Entiat is listed as a "category 5" impaired water body by WDOE, and this listing was approved by the U.S. Environmental Protection Agency on January 29, 2009. A category 5 listing requires the state to prepare a Total Maximum Daily Load (TMDL) analysis, with the goal of ensuring that the impaired water body will attain water quality standards (WDOE 2009). In addition to $\mathrm{pH}$, the Entiat River is also listed in the 2008 WDOE Water Quality Assessment as a "category 4B" impaired water body for water temperature. A category 4B listing recognizes a water quality problem that is being solved by a pollution control program; therefore a TMDL analysis is not required (WDOE 2009).

Within this context, The U.S. Forest Service, PNW Research Station, in partnership with ISEMP, is testing an approach to subbasin-scale status and trend monitoring of fundamental water quality parameters of interest to salmon recovery $(\mathrm{pH}$, water temperature, dissolved oxygen, and specific conductivity) in the Entiat River subbasin. The value of these data sets will increase and conclusions will become more robust as the length of record increases.

\section{Methods and materials}

\section{Project area}

The Entiat River drains approximately 1100 square kilometers (425 square miles) of the eastern slope of the central Cascade Mountains in Washington State, and is a tributary to the Columbia 
River. The Entiat is 85 km (53 mi) long, and is located 55 km (34 mi) northwest of Wenatchee. Wildfire, flooding, mass soil and debris movement, and land use have been primary historic disturbance processes. Land use has included floodplain and river channel modification projects and structures, grazing, roading, agriculture, timber harvesting, transport of logs within the river channel, dams for log storage ponds and hydropower generation, residential development, and recreation (CCCD 2004).

\section{$\underline{\text { Site Selection }}$}

Following field reconnaissance and examination of drainage network maps, measurement sites were collocated with the existing network of stream gages. This distribution was consistent with the USGS, National Water Quality Assessment Program approach of making intensive measurements at a few points that integrate critical drainage areas, rather than randomly selecting measurement sites. One water-quality instrument was deployed at each stream-gaging site (Table 1). Instruments were secured in the channel inside protective, $10 \mathrm{~cm}$ (four inch)diameter pipes or other robust enclosure. A $60 \mathrm{~cm}$-long section of perforated pipe was attached to the primary pipe to ensure ample water circulation around the instrument sensors.

\section{$\underline{\text { Instrumentation }}$}

We employed In-Situ, Troll $9500^{1}$ multiparameter, data-logging instruments with a separate sensor for each measured water-quality parameter. All sensors used an EPA approved or recommended methodology (Table 2). Four instruments were deployed during the spring 2008, and were programmed to record data at 15-minute intervals. Instruments were removed during winter periods to avoid damage from river ice and redeployed the following spring as soon as ice conditions allowed.

Table 1. Location of water quality instruments in the Entiat River subbasin.

\begin{tabular}{|c|c|c|c|c|}
\hline River Gage (short name) & Gaging Agency & Gage ID & $\begin{array}{l}\text { River Mile } \\
\text { (RM) }\end{array}$ & $\begin{array}{l}\text { Coordinates } \\
\text { (Lat., Long.) }\end{array}$ \\
\hline $\begin{array}{l}\text { Entiat River near Entiat } \\
\text { ('Entiat') }\end{array}$ & USGS & 12452990 & 1.4 & $\begin{array}{l}47^{\circ} 39^{\prime} 48^{\prime \prime}, \\
120^{\circ} 14^{\prime} 58^{\prime \prime}\end{array}$ \\
\hline $\begin{array}{l}\text { Mad River at Ardenvoir } \\
\text { ('Mad') }\end{array}$ & USGS & 12452800 & 0.4 & $\begin{array}{l}47^{\circ} 44^{\prime} 13^{\prime \prime}, \\
120^{\circ} 22^{\prime} 03^{\prime \prime}\end{array}$ \\
\hline $\begin{array}{l}\text { Entiat River near Ardenvoir } \\
\text { ('Ardenvoir') }\end{array}$ & USGS & 12452890 & 18 & $\begin{array}{l}47^{\circ} 49^{\prime} 07^{\prime \prime}, \\
120^{\circ} 25^{\prime} 19^{\prime \prime}\end{array}$ \\
\hline $\begin{array}{l}\text { Entiat River at North Fork } \\
\text { Campground ('North Fork') }\end{array}$ & $\mathrm{WDOE}^{2} / \mathrm{CCD}^{3}$ & 46A170 & 34 & $\begin{array}{l}47^{\circ} 59^{\prime} 15^{\prime \prime}, \\
120^{\circ} 34^{\prime} 47^{\prime \prime}\end{array}$ \\
\hline
\end{tabular}

\footnotetext{
${ }^{1}$ The use of trade names in this paper is for the information and convenience of the reader. Such use does not constitute and official endorsement by the U.S. Department of Agriculture of any product to the exclusion of other that may be suitable.

${ }^{2}$ Washington State Department of Ecology

${ }^{3}$ Cascadia Conservation District
} 

Table 2. Sensor methodology and manufacturer's stated accuracy (In-Situ 2008)

\begin{tabular}{|l|l|l|}
\hline \multicolumn{1}{|c|}{ Variable } & \multicolumn{1}{|c|}{ Methodology } & \multicolumn{1}{c|}{ Accuracy } \\
\hline $\mathrm{pH}$ & Standard Methods 4500-H+, EPA 150.2 & $0.1 \mathrm{pH}$ units \\
\hline Temperature & EPA 170.1 & $0.1^{\circ} \mathrm{C}$ \\
\hline DO & $\begin{array}{c}\text { Optical DO sensors -- ASTM \#D888-05, Method C } \\
\text { Note: EPA has recommended to each of its } \\
\text { Regions Interim Approval for the use of this } \\
\text { method. 40 CFR part 136.5 (ATP Case No. } \\
\text { N05-0046). }\end{array}$ & $0.2 \mathrm{mg} / \mathrm{L}$ \\
\hline $\begin{array}{l}\text { Specific } \\
\text { Conductivity }\end{array}$ & Standard Methods 2510, EPA 120.1 & $0.5 \%$ or $2 \mu \mathrm{S} / \mathrm{cm}$ \\
\hline
\end{tabular}

QA/QC testing with a reference instrument Instrument installation was followed by a period lasting approximately one month of very frequent field visits to check and adjust instrument function and calibration. Following this initial period, instruments and batteries were inspected and data downloaded at one to two week intervals. During each site visit a fifth instrument, calibrated before and after use, was used as a portable, hand-held reference for checking field instrument data. Field-deployed sensors were recalibrated whenever deviation from reference data exceeded a predefined standard (Table 3).

Table 3. Maximum acceptable field vs. reference deviations

\begin{tabular}{|l|l|}
\hline \multicolumn{1}{|c|}{ Variable } & \multicolumn{1}{c|}{ Threshold deviation } \\
\hline $\mathrm{pH}$ & $0.2 \mathrm{pH}$ units \\
\hline Temperature & $0.5^{\circ} \mathrm{C}$ \\
\hline $\mathrm{DO}$ & $1 \mathrm{mg} / \mathrm{L}$ \\
\hline Conductivity & $5 \mu \mathrm{S} / \mathrm{cm}$ \\
\hline
\end{tabular}

Reference test procedure:

1. The reference instrument was allowed to equilibrate to the stream water environment for a minimum of five minutes.

2. Field and reference instrument readings were recorded and compared.

3. If the difference between the field and reference readings was greater than the threshold deviation (Table 3), then the field sensor was recalibrated.

4. In addition, sensor calibration was done under the following conditions:

a. Consistent, but within threshold, deviation from the reference for a sustained period

b. Disturbance to the instrument, e.g. sedimentation or biofouling

c. Sensor performance outside manufacturer's recommendation

\section{Calibration}

Instruments were designed to accommodate field calibration at ambient, on-site temperature, elevation, and barometric pressure, thereby improving accuracy of DO saturation measurements 
relative to calibration at an off-site lab. Because these instruments remained on site during calibration, interruption of data collection was minimized. All calibrations and testing were done using the manufacturer's software and specifications, thereby meeting or exceeding the manufacturer's QA/QC guidelines (In-Situ 2009).

$\mathrm{pH}:$

A two-point $\mathrm{pH}$ calibration was done using $\mathrm{pH} 7$ and 10 buffer solutions, thereby spanning the expected range of $\mathrm{pH}$ in the Entiat and Mad Rivers. The reference sensor was calibrated in the lab before each day of field checks, and a one-point pH 7 check was done at the end of the day. If this reading deviated from the standard by more than 0.2 $\mathrm{pH}$ units (the threshold deviation), then the reference readings for that day were not used. If the $\mathrm{pH}$ calibration parameters (slope, offset, and response) were outside the manufacturer's recommended range, then the sensor required maintenance or replacement.

\section{Temperature:}

Temperature sensors were not designed for user calibration. Therefore they were replaced if deviations from the reference sensor were not within standards.

\section{Dissolved oxygen:}

A vented cable and integrated temperature sensor allowed the optical DO sensor to incorporate real-time barometric pressure and temperature into the calculation of percent oxygen saturation. Therefore, maximum accuracy was achieved by calibrating both field and reference DO sensors in the field. The reference sensor was calibrated at the approximate median elevation of the field sites (the Ardenvoir gage). Two-point calibrations were done using solutions at $100 \%$ and $0 \%$ saturation. $100 \%$ saturation was obtained by bubbling air through deionized water. A solution of sodium sulfite provided $0 \%$ saturation. Following full calibration, the reference sensor was checked in a $100 \%$ oxygen-saturated solution immediately before and after each cycle of reference checks. If measurements deviated from the standard by more than $10 \%$, then the reference DO sensor was recalibrated in the field and the reference DO readings for that day were not used.

\section{Specific conductivity:}

A one-point specific conductivity calibration was done using a $147 \mu \mathrm{S} / \mathrm{cm}$ standard. The reference sensor was calibrated in the lab before and after each field day. Conductivity readings before and after each calibration were compared to check sensor performance. If end-of-the-day values deviated by more than $5 \mu \mathrm{S} / \mathrm{cm}$, then the reference conductivity readings for that day were not used. If the cell constant $\left(\mathrm{K}_{\text {cell }}\right)$ for the conductivity sensor fell outside the manufacturer's recommended range (0.33 to 0.39 ), then the sensor required maintenance or replacement.

\section{Data Processing}

Time series of instantaneous data measured at 15-minute intervals and longer-term means or medians $(\mathrm{pH})$ were examined to investigate short- and long-term temporal trends and the timing and longevity of water quality extremes. Spatial variation was interpreted by comparing results 
across the array of instrumented sites. Data of questionable quality included extreme outliers, erratic fluctuations, or extreme variability relative to surrounding data, and occurred as a result of ice effects, biofouling, or other factors. Such occurrences were flagged for detailed scrutiny. Data determined to be unreliable were treated as missing, and not included in reporting or analyses. However, data corrected for ordinary instrument drift, as determined through reference tests and field calibrations, were considered valid, first-quality data following Carroll et al. (2006). All data, regardless of designations or adjustments, were recorded by category in the database along with notes regarding reference checks, calibrations, unreliable data, or other relevant issues.

\section{Results}

$\mathrm{pH}$

In 2008 at all sites the highest median-monthly and median-daily $\mathrm{pH}$ values occurred during either the March-April or August-September period. Both periods are characterized by relatively low discharge and high exposure to solar radiation. A sharp drop in $\mathrm{pH}$ occurred during the high-flow, snowmelt runoff period from late spring through early summer (Table 4, Fig. 1). Median-daily $\mathrm{pH}$ was generally similar at the North Fork and Ardenvoir gages, suggesting little change in the intervening 16 river miles. Similarly, during the early spring, prior to high snowmelt runoff, median-daily $\mathrm{pH}$ tended to be similar at the Entiat and Mad River gages, where it was consistently greater than at Ardenvoir or North Fork (Fig. 1). Following the snowmelt peak runoff period, median-daily $\mathrm{pH}$ at the Mad River gage tended to be higher than at the Entiat gage, where $\mathrm{pH}$ tended to be higher in the spring than during the summer-fall period. At the other gages, median-daily $\mathrm{pH}$ before the spring snowmelt differed little from that afterward, despite higher summer and fall water temperatures (Fig. 1).

\section{Water temperature}

In 2008 water temperature began rapidly increasing during the receding limb of the annual snowmelt hydrograph. The highest mean-monthly and mean daily temperatures occurred in July and August (Table 4, Fig. 2). Overall, the highest temperatures were measured at the Entiat gage, where mean-daily temperatures exceeded $20^{\circ} \mathrm{C}$ during one brief period in August (Fig. 2). Temperature at the Ardenvoir gage tended to be similar to that at the Mad River gage, although the rapid summertime temperature increase began earlier in the Mad River. At all sites, temperatures began to decrease in late August or early September, and fell to nearly $0^{\circ} \mathrm{C}$ by December. Temperature was consistently lowest at North Fork, the farthest upstream gage (Table 4, Fig. 2).

\section{Dissolved oxygen}

Dissolved oxygen (DO) concentration is inversely related to water temperature. Accordingly, at all measurement sites the lowest concentrations were measured during the period from July through September, the months with highest water temperature and high biologic respiration (Table 4, Fig. 3). Mean-daily DO less than $9 \mathrm{mg} / \mathrm{l}$ was unusual, but occurred at all sites other than the North Fork gage during one brief period in August. At the Entiat gage, mean-daily DO very briefly dropped below $8 \mathrm{mg} / \mathrm{l}$ during this same period (Fig. 3). Mean-daily DO concentration, expressed in percent saturation, remained high throughout the data collection period, never falling below $87 \%$ at any of the sites. Values less than $93 \%$ were unusual, 
Table 4. Monthly means or medians $(\mathrm{pH})$ of water quality parameters. Superscripts denote days of record for incomplete months. Data are presented as follows: $\mathrm{pH}$ (pH units), water temperature (Temp, ${ }^{\circ} \mathrm{C}$ ), dissolved oxygen (DO, mg/l), specific conductivity (SC, $\mu \mathrm{S} / \mathrm{cm})$

\begin{tabular}{|c|c|c|c|c|c|c|c|c|c|c|c|c|c|c|c|c|}
\hline \multirow[t]{2}{*}{ Month } & \multicolumn{4}{|c|}{ Entiat } & \multicolumn{4}{|c|}{ Mad } & \multicolumn{4}{|c|}{ Ardenvoir } & \multicolumn{4}{|c|}{ North Fork } \\
\hline & $\mathrm{pH}$ & Temp & $\mathrm{DO}$ & SC & $\mathrm{pH}$ & Temp & $\mathrm{DO}$ & SC & $\mathrm{pH}$ & Temp & $\mathrm{DO}$ & SC & $\mathrm{pH}$ & Temp & $\mathrm{DO}$ & SC \\
\hline Mar-08 & $8.05^{i}$ & $4.82^{i}$ & $12.71^{i}$ & $128.2^{f}$ & $8.10^{\prime}$ & $2.87^{1}$ & $12.86^{\prime}$ & $144.0^{j}$ & $7.50^{\prime}$ & $3.60^{\prime}$ & $11.82^{\prime}$ & $56.97^{\prime}$ & $7.42^{i}$ & $1.06^{i}$ & $12.16^{i}$ & $48.09 i$ \\
\hline Apr-08 & 8.16 & 7.75 & 11.79 & 115.5 & 8.00 & 5.15 & 12.17 & $133.4^{t}$ & 7.36 & 5.80 & 11.38 & 53.42 & 7.40 & 2.53 & 11.95 & 46.02 \\
\hline Jun-08 & 7.50 & 9.67 & 11.18 & 38.99 & 7.51 & 8.49 & 11.25 & 46.77 & 7.12 & 7.92 & 11.10 & 32.79 & 7.12 & 5.72 & 11.46 & 32.11 \\
\hline Jul-08 & 7.72 & 15.29 & 9.76 & 52.01 & 7.76 & 14.20 & 9.77 & 76.54 & 7.34 & 12.60 & 9.86 & 38.00 & 7.23 & 9.02 & 10.58 & 34.40 \\
\hline Aug-08 & 7.80 & 17.30 & 9.05 & 81.74 & 8.03 & 14.79 & 9.65 & 106.0 & 7.47 & 14.61 & 9.25 & 47.30 & 7.48 & 10.22 & 10.18 & 40.52 \\
\hline Nov-08 & 7.48 & 4.15 & 12.28 & 76.68 & 7.77 & 3.14 & 12.58 & 92.08 & 7.33 & 3.16 & 12.00 & 45.60 & 7.40 & 1.96 & 11.99 & 41.17 \\
\hline Dec-08 & $7.58^{\mathrm{b}}$ & $3.67^{b}$ & $12.61^{b}$ & $74.07^{b}$ & $7.78^{\mathrm{b}}$ & $2.66^{\mathrm{b}}$ & $12.75^{\mathrm{b}}$ & $87.09^{b}$ & $7.42^{\mathrm{b}}$ & $2.60^{\mathrm{b}}$ & $12.16^{\mathrm{b}}$ & $46.57^{b}$ & $7.42^{\mathrm{b}}$ & $1.90^{\mathrm{b}}$ & $11.96^{\mathrm{b}}$ & $42.40^{\mathrm{b}}$ \\
\hline
\end{tabular}




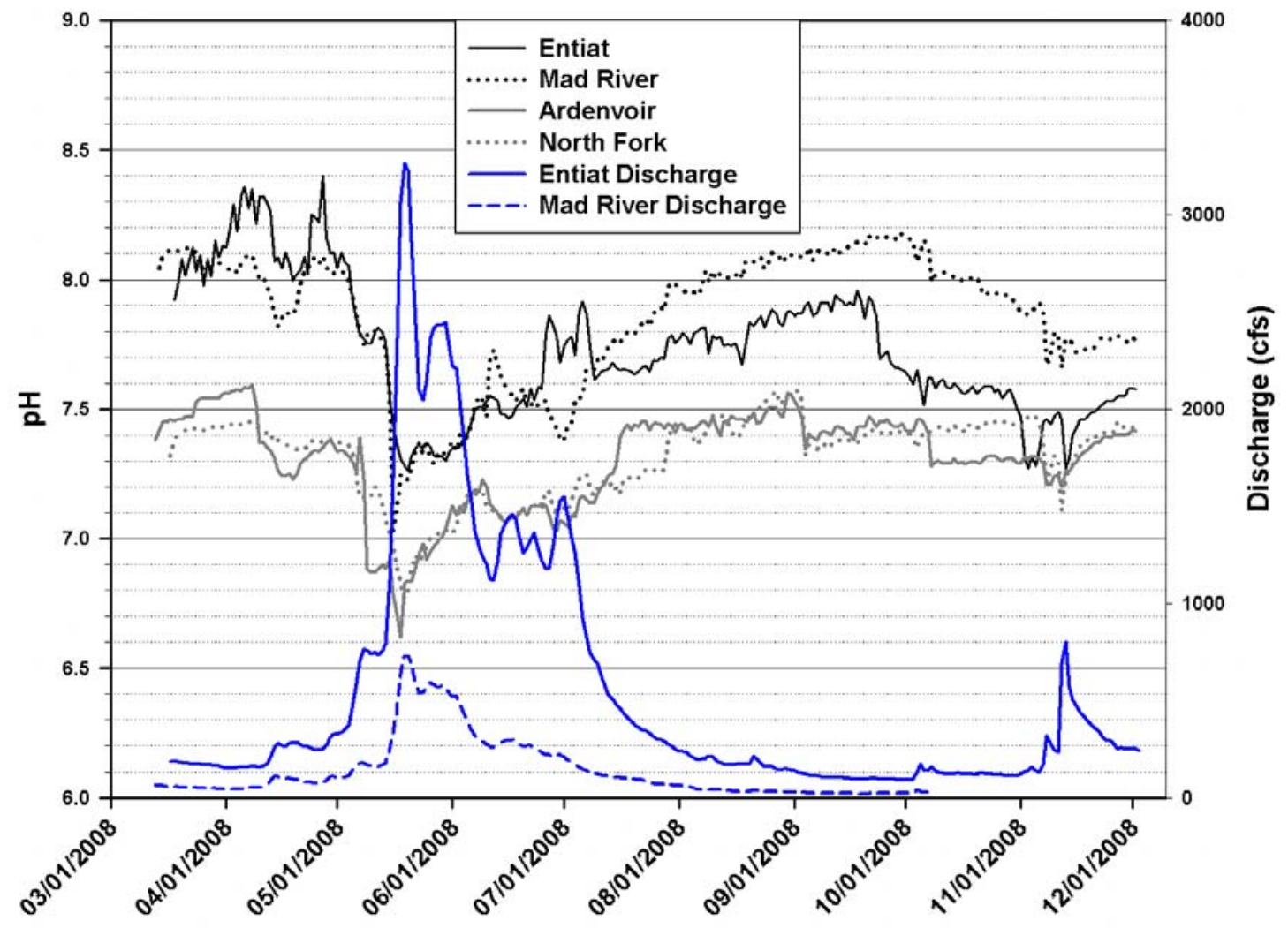

Figure 1. Median-daily $\mathrm{pH}$.

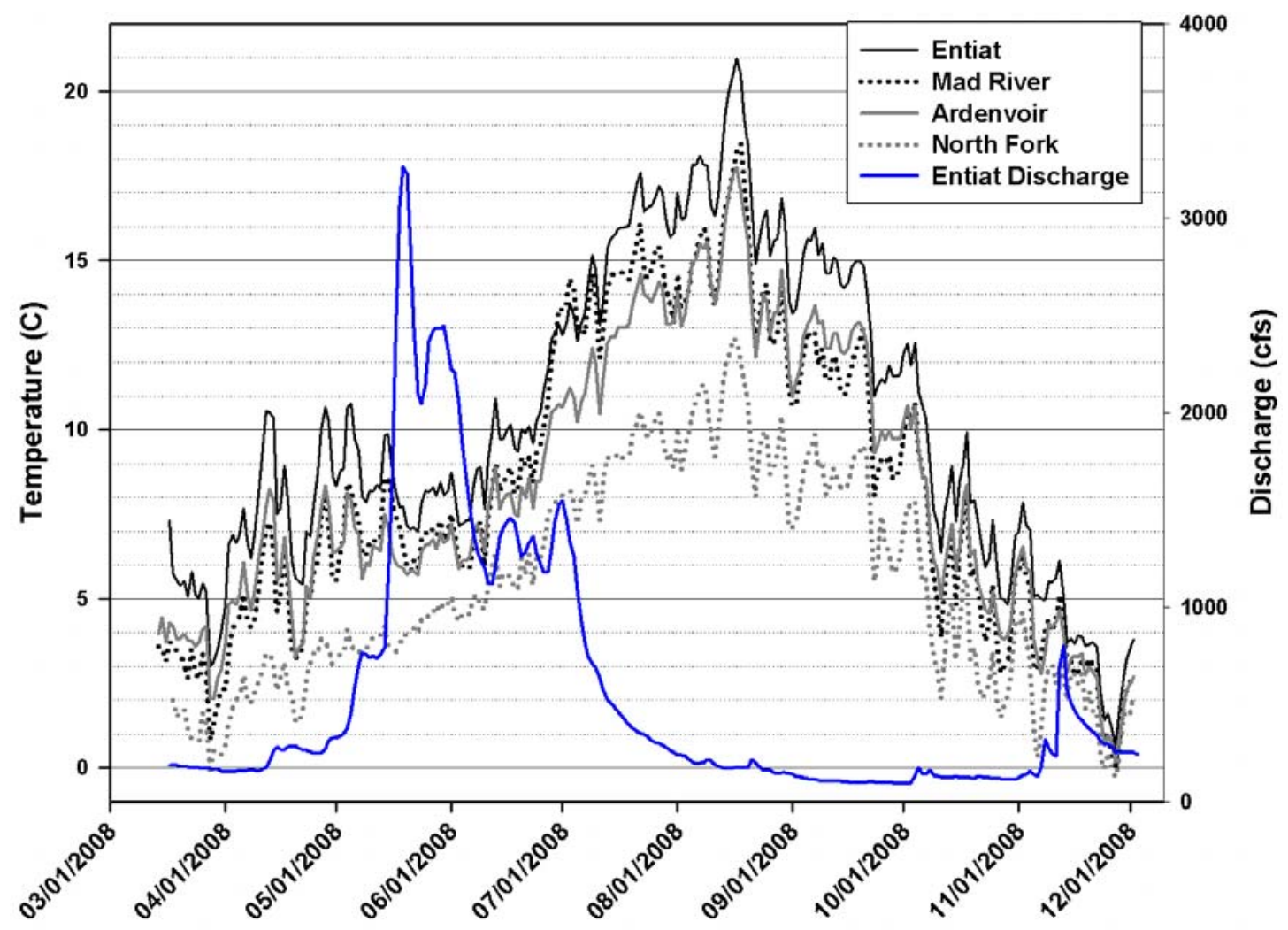

Figure 2. Mean-daily water temperature. 


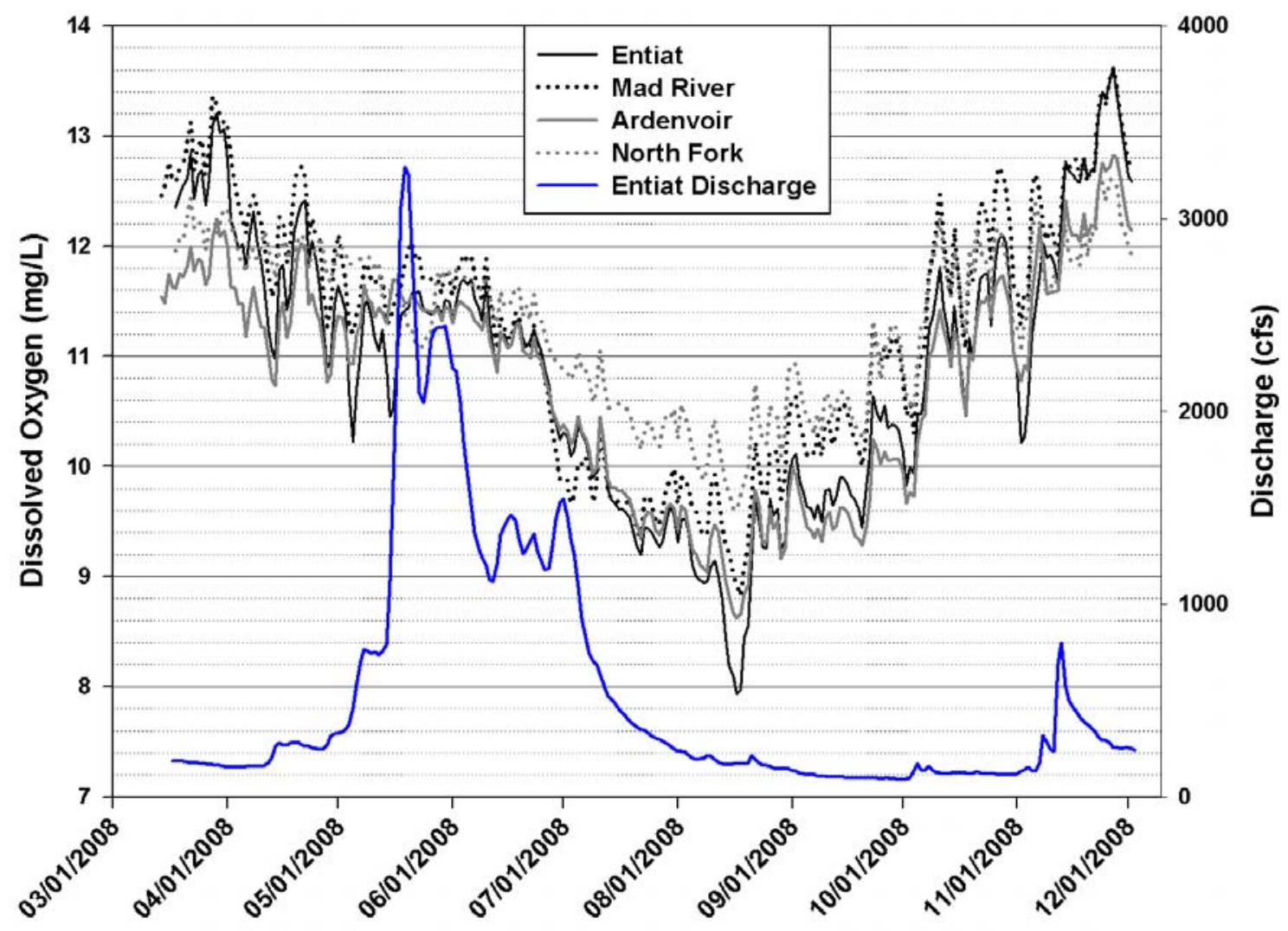

Figure 3. Mean-daily dissolved oxygen.

occurring only a few days each year at all sites except the Mad River gage, where the lowest measurement was $95 \%$.

\section{Specific conductivity}

As with pH, specific conductivity (SC) decreased sharply during the annual snowmelt hydrograph. Accordingly, the lowest mean-monthly values occurred in May and June at all sites (Table 4).

Highest mean-daily SC occurred in the Mad River, where values exceeded $150 \mu$ S/cm (Fig. 4). At the Entiat gage mean-daily specific conductivities were only slightly lower than at the Mad River gage, but otherwise similar. Mean-daily specific conductivities at the Ardenvoir and North Fork gages were markedly lower than at the Mad and Entiat gages. Values were generally lowest at the North Fork gage. Daily means did not exceed $60 \mu \mathrm{S} / \mathrm{cm}$ at Ardenvoir or $50 \mu \mathrm{S} / \mathrm{cm}$ at North Fork (Fig. 4). 


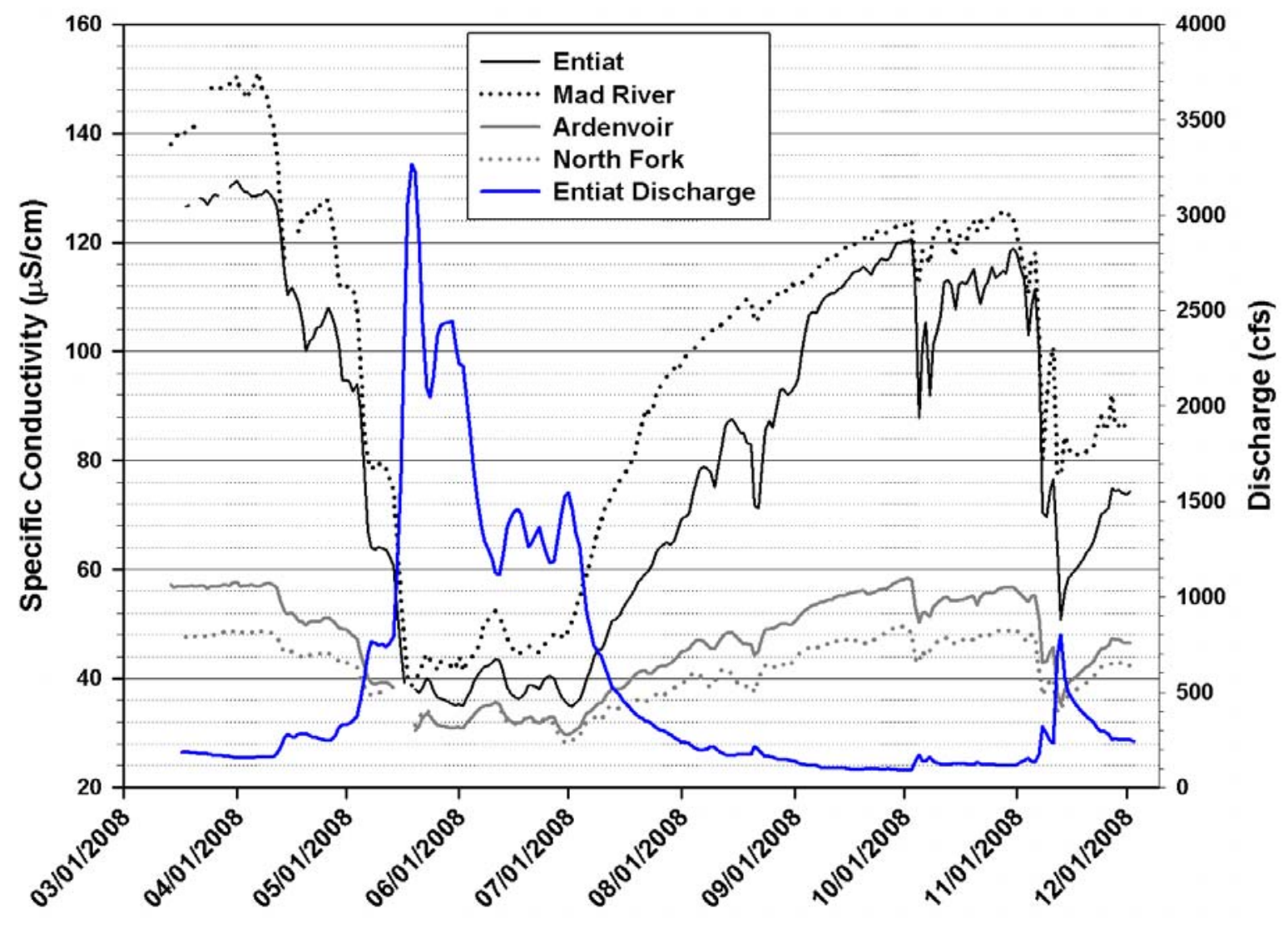

Figure 4. Mean-daily specific conductivity. 


\section{References}

BPA. 2008. Bonneville Power Administration, Integrated Fish and Wildlife Program. (November 2008; http://www.efw.bpa.gov/IntegratedFWP/)

Carroll J, O’Neal S, Golding S. 2006. Wenatchee River basin dissolved oxygen, pH, and phosphorus total maximum daily load study, Publication Number 06-03-018. Olympia, Washington: Washington State Department of Ecology.

CCCD. 2004. Entiat Water Resource Inventory Area (WRIA) 46 Management Plan, prepared for the Entiat Watershed Planning Unit. Wenatchee, WA: Chelan County Conservation District, 13 Chapters, plus appendices.

In-Situ. 2008. In-Situ, Inc. (November 2008; http://www.in-situ.com)

ISEMP. 2008. Integrated Status and Effectiveness Monitoring Program. (November 2008; http://www.nwfsc.noaa.gov/research/divisions/cbd/mathbio/isemp/ index.cfm)

UCSRB. 2007. Upper Columbia Spring Chinook Salmon and Steelhead Recovery Plan, http://www.ucsrb.com/UCSRP\%20Final\%209-13-2007.pdf. Wenatchee, WA: Upper Columbia Salmon Recovery Board. 307 pp. + App.

WDOE. 2009. Water Quality Assessment Process. February 2009, http://www.ecy.wa.gov/programs/wq/303d/2008/index.html 


\section{Appendices}

Appendix A. Daily medians (pH) or means of water quality parameters at the Entiat gage. Discharge is from the Entiat gage.

\begin{tabular}{|c|c|c|c|c|c|}
\hline 3/17/08 & --- & --- & --- & --- & 187 \\
\hline 3/18/08 & 7.92 & 5.8 & 12.36 & 126.54 & 188 \\
\hline 3/19/08 & 7.98 & 5.5 & 12.47 & 126.81 & 186 \\
\hline $3 / 20 / 08$ & 8.08 & 5.4 & 12.55 & --- & 184 \\
\hline $3 / 21 / 08$ & 8.02 & 5.5 & 12.61 & --- & 180 \\
\hline $3 / 22 / 08$ & 8.07 & 5.1 & 12.85 & 128.08 & 177 \\
\hline 3/23/08 & 8.13 & 5.8 & 12.43 & 127.77 & 176 \\
\hline $3 / 24 / 08$ & 8.03 & 5.1 & 12.65 & 126.93 & 176 \\
\hline $3 / 25 / 08$ & 8.10 & 5.0 & 12.69 & 128.06 & 172 \\
\hline $3 / 26 / 08$ & 7.98 & 5.5 & 12.37 & 128.71 & 170 \\
\hline $3 / 27 / 08$ & 8.08 & 5.2 & 12.61 & 128.51 & 168 \\
\hline 3/28/08 & 8.01 & 3.0 & 13.09 & --- & 169 \\
\hline $3 / 29 / 08$ & 8.15 & 3.1 & 13.20 & --- & 164 \\
\hline 3/30/08 & 8.09 & 3.5 & 13.03 & 130.13 & 164 \\
\hline 3/31/08 & 8.13 & 4.0 & 13.05 & 130.74 & 158 \\
\hline 4/1/08 & 8.13 & 4.8 & 12.78 & 131.27 & 154 \\
\hline 4/2/08 & 8.19 & 6.6 & 12.23 & 130.29 & 155 \\
\hline $4 / 3 / 08$ & 8.29 & 6.9 & 12.11 & 129.26 & 154 \\
\hline 4/4/08 & 8.19 & 6.7 & 11.97 & 129.17 & 156 \\
\hline 4/5/08 & 8.32 & 6.9 & 12.02 & 128.42 & 156 \\
\hline 4/6/08 & 8.36 & 7.7 & 11.79 & 128.44 & 158 \\
\hline 4/7/08 & 8.28 & 6.7 & 12.09 & 128.77 & 162 \\
\hline 4/8/08 & 8.35 & 6.2 & 12.32 & 128.76 & 161 \\
\hline 4/9/08 & 8.22 & 6.9 & 12.05 & 129.44 & 159 \\
\hline 4/10/08 & 8.32 & 8.1 & 11.86 & 129.05 & 158 \\
\hline 4/11/08 & 8.32 & 9.2 & 11.63 & 127.98 & 164 \\
\hline $4 / 12 / 08$ & 8.30 & 10.5 & 11.24 & 126.63 & 176 \\
\hline 4/13/08 & 8.27 & 10.5 & 11.06 & 122.48 & 207 \\
\hline 4/14/08 & 8.07 & 10.3 & 10.97 & 114.70 & 259 \\
\hline 4/15/08 & 8.09 & 7.5 & 11.79 & 110.44 & 280 \\
\hline 4/16/08 & 8.05 & 7.7 & 11.83 & 111.75 & 268 \\
\hline $4 / 17 / 08$ & 8.11 & 8.9 & 11.42 & 110.56 & 266 \\
\hline 4/18/08 & 8.06 & 7.9 & 11.58 & 109.02 & 281 \\
\hline 4/19/08 & 8.00 & 6.1 & 12.11 & 105.40 & 282 \\
\hline 4/20/08 & 8.03 & 5.6 & 12.27 & 100.09 & 285 \\
\hline 4/21/08 & 8.04 & 5.5 & 12.39 & 102.06 & 274 \\
\hline $4 / 22 / 08$ & 8.09 & 5.4 & 12.42 & 102.48 & 265 \\
\hline $4 / 23 / 08$ & 8.02 & 7.0 & 11.85 & 104.34 & 264 \\
\hline 4/24/08 & 8.25 & 6.8 & 12.05 & 104.64 & 257 \\
\hline $4 / 25 / 08$ & 8.24 & 7.8 & 11.86 & 105.90 & 249 \\
\hline 4/26/08 & 8.22 & 8.7 & 11.63 & 107.91 & 246 \\
\hline $4 / 27 / 08$ & 8.40 & 10.0 & 11.21 & 106.71 & 252 \\
\hline 4/28/08 & 8.16 & 10.7 & 10.90 & 104.70 & 268 \\
\hline 4/29/08 & 8.10 & 10.2 & 10.95 & 101.35 & 315 \\
\hline 4/30/08 & 8.11 & 8.6 & 11.47 & 94.77 & 327 \\
\hline 5/1/08 & 8.05 & 8.3 & 11.64 & 94.74 & 327 \\
\hline
\end{tabular}




\begin{tabular}{|c|c|c|c|c|c|}
\hline $5 / 2 / 08$ & 8.11 & 8.8 & 11.52 & 94.46 & 338 \\
\hline $5 / 3 / 08$ & 8.07 & 8.8 & 11.41 & 92.58 & 351 \\
\hline $5 / 4 / 08$ & 8.05 & 10.6 & 10.68 & 94.00 & 377 \\
\hline $5 / 5 / 08$ & 7.93 & 10.8 & 10.22 & 89.29 & 461 \\
\hline $5 / 6 / 08$ & 7.85 & 9.7 & 10.70 & 77.44 & 585 \\
\hline $5 / 7 / 08$ & 7.79 & 9.4 & 11.03 & 67.14 & 701 \\
\hline $5 / 8 / 08$ & 7.77 & 8.0 & 11.45 & 63.92 & 767 \\
\hline $5 / 9 / 08$ & 7.76 & 7.9 & 11.51 & 63.66 & 757 \\
\hline 5/10/08 & 7.76 & 8.2 & 11.35 & 64.17 & 743 \\
\hline 5/11/08 & 7.80 & 8.2 & 11.15 & 63.85 & 752 \\
\hline $5 / 12 / 08$ & 7.82 & 8.4 & 11.05 & 63.60 & 736 \\
\hline $5 / 13 / 08$ & 7.80 & 8.2 & 11.23 & 62.39 & 752 \\
\hline $5 / 14 / 08$ & 7.76 & 9.8 & 10.90 & 60.82 & 794 \\
\hline 5/15/08 & 7.54 & 9.9 & 10.45 & 53.22 & 1123 \\
\hline $5 / 16 / 08$ & 7.44 & 8.8 & 10.53 & 44.97 & 1647 \\
\hline $5 / 17 / 08$ & 7.36 & 8.2 & 11.06 & 39.21 & 2366 \\
\hline $5 / 18 / 08$ & 7.31 & 7.7 & 11.37 & --- & 3065 \\
\hline 5/19/08 & --- & 7.7 & 11.42 & --- & 3264 \\
\hline $5 / 20 / 08$ & 7.26 & 7.1 & 11.44 & 38.07 & 3227 \\
\hline $5 / 21 / 08$ & 7.32 & 7.1 & 11.57 & 37.40 & 2861 \\
\hline $5 / 22 / 08$ & 7.35 & 7.1 & 11.58 & 38.28 & 2434 \\
\hline $5 / 23 / 08$ & 7.36 & 7.0 & 11.59 & 39.95 & 2098 \\
\hline $5 / 24 / 08$ & 7.35 & 7.8 & 11.43 & 39.57 & 2045 \\
\hline $5 / 25 / 08$ & 7.37 & 8.2 & 11.40 & 37.94 & 2147 \\
\hline $5 / 26 / 08$ & 7.35 & 8.1 & 11.38 & 36.57 & 2369 \\
\hline $5 / 27 / 08$ & 7.32 & 8.3 & 11.39 & 36.33 & 2421 \\
\hline $5 / 28 / 08$ & 7.32 & 8.1 & 11.45 & 36.14 & 2436 \\
\hline $5 / 29 / 08$ & 7.32 & 8.5 & 11.36 & 35.80 & 2433 \\
\hline $5 / 30 / 08$ & 7.30 & 8.1 & 11.51 & 35.29 & 2443 \\
\hline $5 / 31 / 08$ & 7.33 & 8.2 & 11.49 & 35.17 & 2324 \\
\hline 6/1/08 & 7.35 & 8.8 & 11.32 & 35.34 & 2221 \\
\hline $6 / 2 / 08$ & 7.35 & 8.1 & 11.49 & 34.99 & 2208 \\
\hline $6 / 3 / 08$ & 7.36 & 7.2 & 11.66 & 36.38 & 2048 \\
\hline $6 / 4 / 08$ & 7.39 & 7.2 & 11.69 & 37.75 & 1842 \\
\hline $6 / 5 / 08$ & 7.41 & 7.3 & 11.65 & 39.42 & 1658 \\
\hline 6/6/08 & 7.45 & 7.3 & 11.70 & 40.47 & 1518 \\
\hline $6 / 7 / 08$ & 7.50 & 8.4 & 11.53 & 41.45 & 1374 \\
\hline 6/8/08 & 7.51 & 8.8 & 11.45 & 42.17 & 1292 \\
\hline $6 / 9 / 08$ & 7.51 & 8.9 & 11.32 & 42.49 & 1242 \\
\hline 6/10/08 & 7.53 & 7.7 & 11.67 & 42.80 & 1201 \\
\hline 6/11/08 & 7.55 & 9.1 & 11.30 & 43.57 & 1127 \\
\hline 6/12/08 & 7.55 & 10.0 & 11.09 & 43.33 & 1117 \\
\hline 6/13/08 & 7.54 & 10.9 & 10.85 & 41.34 & 1209 \\
\hline 6/14/08 & 7.48 & 9.7 & 11.18 & 38.41 & 1360 \\
\hline 6/15/08 & 7.48 & 9.7 & 11.20 & 37.45 & 1407 \\
\hline 6/16/08 & 7.47 & 10.0 & 11.10 & 36.79 & 1441 \\
\hline 6/17/08 & 7.48 & 10.1 & 11.09 & 36.35 & 1460 \\
\hline 6/18/08 & 7.51 & 9.4 & 11.31 & 36.44 & 1433 \\
\hline 6/19/08 & 7.52 & 9.4 & 11.33 & 37.43 & 1344 \\
\hline 6/20/08 & 7.54 & 10.0 & 11.15 & 38.87 & 1261 \\
\hline 6/21/08 & 7.51 & 9.9 & 11.08 & 38.87 & 1288 \\
\hline
\end{tabular}




\begin{tabular}{|c|c|c|c|c|c|}
\hline $6 / 22 / 08$ & 7.58 & 10.1 & 11.14 & 38.41 & 1329 \\
\hline 6/23/08 & 7.54 & 9.6 & 11.25 & 38.03 & 1364 \\
\hline $6 / 24 / 08$ & 7.59 & 10.2 & 11.14 & 39.51 & 1279 \\
\hline 6/25/08 & 7.58 & 10.6 & 11.04 & 40.26 & 1221 \\
\hline 6/26/08 & 7.79 & 11.2 & 10.88 & 40.50 & 1178 \\
\hline $6 / 27 / 08$ & 7.86 & 11.8 & 10.74 & 40.00 & 1184 \\
\hline $6 / 28 / 08$ & 7.82 & 12.7 & 10.50 & 38.32 & 1300 \\
\hline 6/29/08 & 7.78 & 12.8 & 10.40 & 36.59 & 1439 \\
\hline 6/30/08 & 7.68 & 13.1 & 10.24 & 35.95 & 1527 \\
\hline $7 / 1 / 08$ & 7.75 & 12.8 & 10.30 & 35.16 & 1548 \\
\hline $7 / 2 / 08$ & 7.77 & 13.1 & 10.28 & 34.78 & 1455 \\
\hline 7/3/08 & 7.78 & 13.8 & 10.09 & 35.54 & 1339 \\
\hline $7 / 4 / 08$ & 7.71 & 13.4 & 10.15 & 36.18 & 1260 \\
\hline $7 / 5 / 08$ & 7.88 & 12.6 & 10.39 & 37.76 & 1089 \\
\hline $7 / 6 / 08$ & 7.92 & 13.2 & 10.29 & 39.91 & 927 \\
\hline $7 / 7 / 08$ & 7.87 & 13.4 & 10.22 & 41.62 & 822 \\
\hline 7/8/08 & 7.74 & 14.3 & 10.08 & 43.98 & 746 \\
\hline $7 / 9 / 08$ & 7.62 & 15.2 & 9.89 & 45.29 & 709 \\
\hline $7 / 10 / 08$ & 7.63 & 14.7 & 9.95 & 45.31 & 689 \\
\hline 7/11/08 & 7.65 & 13.1 & 10.43 & 46.59 & 634 \\
\hline 7/12/08 & 7.65 & 13.8 & 10.22 & 48.94 & 574 \\
\hline 7/13/08 & 7.66 & 15.3 & 9.80 & 50.58 & 526 \\
\hline 7/14/08 & 7.68 & 15.7 & 9.70 & 50.97 & 508 \\
\hline $7 / 15 / 08$ & 7.66 & 15.7 & 9.67 & 51.60 & 484 \\
\hline $7 / 16 / 08$ & 7.65 & 16.0 & 9.61 & 52.90 & 459 \\
\hline $7 / 17 / 08$ & 7.66 & 16.0 & 9.62 & 54.05 & 438 \\
\hline 7/18/08 & 7.65 & 16.0 & 9.57 & 55.08 & 414 \\
\hline 7/19/08 & 7.65 & 16.0 & 9.54 & 56.26 & 396 \\
\hline $7 / 20 / 08$ & 7.64 & 16.7 & 9.40 & 57.55 & 378 \\
\hline 7/21/08 & 7.65 & 17.2 & 9.28 & 58.45 & 362 \\
\hline 7/22/08 & 7.66 & 17.6 & 9.20 & 59.10 & 351 \\
\hline $7 / 23 / 08$ & 7.67 & 16.4 & 9.45 & 59.74 & 345 \\
\hline $7 / 24 / 08$ & 7.65 & 16.6 & 9.44 & 61.11 & 334 \\
\hline $7 / 25 / 08$ & 7.69 & 16.6 & 9.39 & 63.02 & 317 \\
\hline $7 / 26 / 08$ & 7.69 & 16.9 & 9.33 & 64.07 & 305 \\
\hline $7 / 27 / 08$ & 7.70 & 17.2 & 9.27 & 64.51 & 300 \\
\hline $7 / 28 / 08$ & 7.70 & 17.0 & 9.33 & 64.94 & 290 \\
\hline $7 / 29 / 08$ & 7.77 & 16.1 & 9.53 & 64.53 & 277 \\
\hline 7/30/08 & 7.79 & 15.7 & 9.64 & 65.17 & 265 \\
\hline $7 / 31 / 08$ & 7.76 & 15.8 & 9.57 & 67.47 & 252 \\
\hline $8 / 1 / 08$ & 7.77 & 17.0 & 9.31 & 69.21 & 238 \\
\hline $8 / 2 / 08$ & 7.80 & 16.2 & 9.51 & 69.70 & 238 \\
\hline 8/3/08 & 7.78 & 16.2 & 9.52 & 70.24 & 234 \\
\hline $8 / 4 / 08$ & 7.75 & 17.0 & 9.39 & 73.43 & 218 \\
\hline $8 / 5 / 08$ & 7.80 & 17.8 & 9.12 & 76.20 & 204 \\
\hline 8/6/08 & 7.80 & 17.8 & 8.99 & 78.45 & 196 \\
\hline 8/7/08 & 7.81 & 18.1 & 8.97 & 78.75 & 198 \\
\hline 8/8/08 & 7.82 & 17.9 & 8.94 & 78.56 & 200 \\
\hline 8/9/08 & 7.72 & 17.8 & 8.96 & 77.93 & 216 \\
\hline 8/10/08 & 7.79 & 16.6 & 9.08 & 75.08 & 211 \\
\hline 8/11/08 & 7.77 & 16.3 & 9.15 & 78.75 & 193 \\
\hline
\end{tabular}




\begin{tabular}{|c|c|c|c|c|c|}
\hline 8/12/08 & 7.78 & 16.9 & 9.01 & 82.96 & 180 \\
\hline 8/13/08 & 7.75 & 18.4 & 8.78 & 86.45 & 172 \\
\hline 8/14/08 & 7.75 & 19.5 & 8.44 & 87.37 & 169 \\
\hline 8/15/08 & 7.75 & 20.1 & 8.19 & 87.41 & 169 \\
\hline 8/16/08 & 7.76 & 20.6 & 8.11 & 86.42 & 173 \\
\hline 8/17/08 & 7.72 & 21.0 & 7.94 & 85.08 & 176 \\
\hline 8/18/08 & 7.67 & 20.5 & 7.97 & 85.05 & 173 \\
\hline 8/19/08 & 7.76 & 19.2 & 8.45 & 83.24 & 172 \\
\hline 8/20/08 & 7.84 & 18.4 & 8.55 & 82.88 & 175 \\
\hline 8/21/08 & 7.82 & 16.7 & 9.14 & 71.70 & 218 \\
\hline $8 / 22 / 08$ & 7.84 & 14.9 & 9.69 & 71.31 & 200 \\
\hline 8/23/08 & 7.86 & 15.6 & 9.50 & 78.69 & 177 \\
\hline 8/24/08 & 7.82 & 16.3 & 9.27 & 85.53 & 163 \\
\hline 8/25/08 & 7.86 & 16.5 & 9.26 & 87.26 & 163 \\
\hline 8/26/08 & 7.89 & 15.1 & 9.71 & 86.01 & 160 \\
\hline 8/27/08 & 7.87 & 15.6 & 9.55 & 89.71 & 150 \\
\hline 8/28/08 & 7.83 & 15.6 & 9.61 & 92.88 & 146 \\
\hline 8/29/08 & 7.82 & 16.8 & 9.25 & 93.13 & 146 \\
\hline 8/30/08 & 7.88 & 16.1 & 9.33 & 91.95 & 147 \\
\hline 8/31/08 & 7.88 & 13.8 & 9.89 & 92.52 & 145 \\
\hline 9/1/08 & 7.86 & 13.4 & 10.07 & 93.73 & 139 \\
\hline $9 / 2 / 08$ & 7.87 & 13.6 & 10.11 & 95.04 & 131 \\
\hline $9 / 3 / 08$ & 7.87 & 14.4 & 9.88 & 99.76 & 127 \\
\hline $9 / 4 / 08$ & 7.90 & 15.2 & 9.76 & 104.04 & 121 \\
\hline 9/5/08 & 7.91 & 15.7 & 9.63 & 106.72 & 118 \\
\hline $9 / 6 / 08$ & 7.86 & 15.6 & 9.62 & 107.22 & 116 \\
\hline $9 / 7 / 08$ & 7.87 & 16.0 & 9.53 & 107.05 & 115 \\
\hline 9/8/08 & 7.91 & 15.2 & 9.65 & 108.36 & 109 \\
\hline 9/9/08 & 7.91 & 15.5 & 9.50 & 109.73 & 105 \\
\hline 9/10/08 & 7.91 & 14.6 & 9.78 & 110.28 & 106 \\
\hline 9/11/08 & 7.88 & 14.6 & 9.80 & 110.66 & 105 \\
\hline 9/12/08 & 7.94 & 15.1 & 9.65 & 110.76 & 105 \\
\hline 9/13/08 & 7.93 & 15.0 & 9.72 & 111.41 & 105 \\
\hline 9/14/08 & 7.92 & 14.3 & 9.90 & 111.80 & 105 \\
\hline 9/15/08 & 7.90 & 14.2 & 9.90 & 112.71 & 101 \\
\hline 9/16/08 & 7.91 & 14.4 & 9.82 & 113.60 & 99 \\
\hline 9/17/08 & 7.90 & 14.8 & 9.74 & 114.49 & 96 \\
\hline 9/18/08 & 7.96 & 15.0 & 9.70 & 114.64 & 96 \\
\hline 9/19/08 & 7.92 & 15.0 & 9.62 & 114.88 & 96 \\
\hline 9/20/08 & 7.85 & 14.9 & 9.44 & 115.52 & 97 \\
\hline 9/21/08 & 7.94 & 14.1 & 9.81 & 114.79 & 100 \\
\hline 9/22/08 & 7.92 & 12.8 & 10.09 & 114.08 & 101 \\
\hline 9/23/08 & 7.86 & 11.0 & 10.64 & 115.69 & 99 \\
\hline $9 / 24 / 08$ & 7.70 & 11.3 & 10.48 & 116.46 & 98 \\
\hline 9/25/08 & 7.71 & 11.5 & 10.41 & 117.12 & 96 \\
\hline 9/26/08 & 7.73 & 11.4 & 10.56 & 116.67 & 99 \\
\hline 9/27/08 & 7.68 & 11.9 & 10.35 & 117.01 & 98 \\
\hline 9/28/08 & 7.66 & 11.6 & 10.38 & 118.75 & 96 \\
\hline 9/29/08 & 7.66 & 11.6 & 10.37 & 119.87 & 94 \\
\hline 9/30/08 & 7.65 & 11.6 & 10.32 & 119.95 & 91 \\
\hline 10/1/08 & 7.65 & 12.3 & 10.13 & 120.24 & 91 \\
\hline
\end{tabular}




\begin{tabular}{|c|c|c|c|c|c|}
\hline $10 / 2 / 08$ & 7.62 & 12.5 & 9.83 & 120.27 & 92 \\
\hline 10/3/08 & 7.60 & 11.9 & 9.99 & 120.60 & 97 \\
\hline 10/4/08 & 7.65 & 12.6 & 9.93 & 112.89 & 136 \\
\hline 10/5/08 & 7.58 & 11.1 & 10.48 & 87.81 & 173 \\
\hline 10/6/08 & 7.52 & 10.7 & 10.48 & 101.21 & 139 \\
\hline 10/7/08 & 7.62 & 10.3 & 10.71 & 105.38 & 139 \\
\hline 10/8/08 & 7.62 & 8.9 & 11.25 & 91.93 & 162 \\
\hline 10/9/08 & 7.58 & 7.6 & 11.35 & 101.21 & 137 \\
\hline $10 / 10 / 08$ & 7.61 & 7.2 & 11.54 & 103.83 & 131 \\
\hline 10/11/08 & 7.62 & 6.4 & 11.81 & 106.52 & 125 \\
\hline $10 / 12 / 08$ & 7.59 & 7.5 & 11.45 & 112.66 & 122 \\
\hline 10/13/08 & 7.58 & 8.2 & 11.25 & 113.10 & 122 \\
\hline 10/14/08 & 7.60 & 8.9 & 11.02 & 112.04 & 125 \\
\hline 10/15/08 & 7.59 & 7.3 & 11.46 & 107.61 & 130 \\
\hline $10 / 16 / 08$ & 7.57 & 8.4 & 11.07 & 112.34 & 125 \\
\hline $10 / 17 / 08$ & 7.56 & 9.0 & 10.83 & 112.64 & 125 \\
\hline 10/18/08 & 7.57 & 9.9 & 10.45 & 112.39 & 124 \\
\hline 10/19/08 & 7.59 & 7.8 & 11.17 & 113.58 & 122 \\
\hline $10 / 20 / 08$ & 7.56 & 7.9 & 10.95 & 115.04 & 120 \\
\hline 10/21/08 & 7.57 & 7.3 & 11.40 & 111.46 & 132 \\
\hline $10 / 22 / 08$ & 7.59 & 6.5 & 11.70 & 108.67 & 125 \\
\hline 10/23/08 & 7.59 & 5.9 & 11.75 & 111.69 & 122 \\
\hline $10 / 24 / 08$ & 7.59 & 6.2 & 11.74 & 112.75 & 122 \\
\hline $10 / 25 / 08$ & 7.57 & 7.3 & 11.28 & 115.47 & 120 \\
\hline 10/26/08 & 7.58 & 5.9 & 11.85 & 113.58 & 120 \\
\hline $10 / 27 / 08$ & 7.54 & 5.0 & 12.05 & 113.81 & 118 \\
\hline $10 / 28 / 08$ & 7.56 & 5.0 & 12.10 & 114.73 & 116 \\
\hline $10 / 29 / 08$ & 7.58 & 4.8 & 12.03 & 114.34 & 115 \\
\hline 10/30/08 & 7.55 & 5.5 & 11.68 & 118.35 & 115 \\
\hline $10 / 31 / 08$ & 7.50 & 6.9 & 11.04 & 118.85 & 115 \\
\hline 11/1/08 & 7.47 & 7.0 & 10.88 & 117.92 & 122 \\
\hline 11/2/08 & 7.31 & 7.9 & 10.21 & 114.99 & 134 \\
\hline $11 / 3 / 08$ & 7.27 & 7.2 & 10.27 & 113.19 & 141 \\
\hline 11/4/08 & 7.32 & 7.0 & 10.59 & 102.87 & 155 \\
\hline 11/5/08 & 7.28 & 5.1 & 11.20 & 108.39 & 140 \\
\hline 11/6/08 & 7.34 & 5.1 & 11.42 & 111.39 & 133 \\
\hline 11/7/08 & 7.45 & 5.0 & 11.73 & 100.20 & 175 \\
\hline 11/8/08 & 7.46 & 5.0 & 12.02 & 70.43 & 321 \\
\hline 11/9/08 & 7.44 & 5.5 & 11.89 & 69.64 & 279 \\
\hline 11/10/08 & 7.47 & 5.5 & 11.92 & 74.23 & 246 \\
\hline $11 / 11 / 08$ & 7.49 & 5.6 & 11.85 & 76.51 & 235 \\
\hline 11/12/08 & 7.46 & 6.1 & 11.67 & 64.12 & 693 \\
\hline $11 / 13 / 08$ & 7.27 & 5.0 & 12.23 & 50.78 & 800 \\
\hline $11 / 14 / 08$ & 7.31 & 3.7 & 12.77 & 56.03 & 574 \\
\hline $11 / 15 / 08$ & 7.40 & 3.8 & 12.68 & 58.43 & 504 \\
\hline 11/16/08 & 7.43 & 3.7 & 12.66 & 59.10 & 470 \\
\hline $11 / 17 / 08$ & 7.46 & 3.9 & 12.59 & 59.97 & 439 \\
\hline 11/18/08 & 7.46 & 3.9 & 12.58 & 60.79 & 417 \\
\hline 11/19/08 & 7.47 & 3.6 & 12.80 & 61.92 & 393 \\
\hline $11 / 20 / 08$ & 7.49 & 3.7 & 12.62 & 63.03 & 374 \\
\hline 11/21/08 & 7.49 & 3.7 & 12.69 & 63.83 & 357 \\
\hline
\end{tabular}




$\begin{array}{lccccc}11 / 22 / 08 & 7.51 & 3.6 & 12.71 & 65.27 & 340 \\ 11 / 23 / 08 & 7.52 & 2.2 & 13.17 & 67.51 & 312 \\ 11 / 24 / 08 & 7.53 & 1.4 & 13.40 & 70.22 & 295 \\ 11 / 25 / 08 & 7.53 & 1.6 & 13.33 & 70.48 & 294 \\ 11 / 26 / 08 & 7.53 & 1.1 & 13.50 & 71.36 & 280 \\ 11 / 27 / 08 & 7.55 & 0.6 & 13.62 & 74.83 & 252 \\ 11 / 28 / 08 & 7.55 & 1.5 & 13.39 & 74.41 & 260 \\ 11 / 29 / 08 & 7.55 & 2.5 & 13.11 & 74.54 & 252 \\ 11 / 30 / 08 & 7.58 & 3.2 & 12.90 & 73.91 & 257 \\ 12 / 1 / 08 & 7.58 & 3.6 & 12.63 & 73.81 & 253 \\ 12 / 2 / 08 & 7.58 & 3.8 & 12.59 & 74.33 & 250 \\ 12 / 3 / 08 & --- & --- & --- & -- & 243\end{array}$


Appendix B. Daily medians (pH) or means of water quality parameters at the Mad River gage. Discharge is from the Mad River gage.

\begin{tabular}{|c|c|c|c|c|c|}
\hline Date & $\mathrm{pH}$ & Temperature (C) & $\begin{array}{c}\text { Dissolved } \\
\text { Oxygen } \\
(\mathrm{mg} / \mathrm{L})\end{array}$ & $\begin{array}{c}\text { Specific } \\
\text { Conductivity } \\
(\mathrm{mS} / \mathrm{cm})\end{array}$ & Discharge (cfs) \\
\hline 3/13/08 & -- & -- & -- & -- & 65 \\
\hline 3/14/08 & 8.05 & 3.6 & 12.46 & 138.02 & 64 \\
\hline 3/15/08 & 8.09 & 3.5 & 12.54 & 138.59 & 62 \\
\hline 3/16/08 & 8.11 & 3.1 & 12.76 & 140.18 & 58 \\
\hline 3/17/08 & 8.11 & 3.7 & 12.60 & 140.52 & 57 \\
\hline 3/18/08 & 8.11 & 3.7 & 12.59 & 140.04 & 57 \\
\hline 3/19/08 & 8.11 & 3.4 & 12.69 & 140.84 & 56 \\
\hline 3/20/08 & 8.12 & 3.3 & 12.73 & 141.14 & 55 \\
\hline 3/21/08 & 8.12 & 3.1 & 12.90 & 141.47 & 54 \\
\hline 3/22/08 & 8.12 & 2.7 & 13.13 & 142.41 & 52 \\
\hline 3/23/08 & 8.12 & 3.5 & 12.69 & --- & 53 \\
\hline $3 / 24 / 08$ & 8.10 & 2.8 & 12.91 & --- & 52 \\
\hline 3/25/08 & 8.05 & 2.6 & 12.97 & 148.34 & 51 \\
\hline 3/26/08 & 8.05 & 3.3 & 12.65 & 147.72 & 50 \\
\hline $3 / 27 / 08$ & 8.05 & 2.9 & 12.89 & 148.26 & 48 \\
\hline 3/28/08 & 8.04 & 0.8 & 13.36 & 148.26 & 50 \\
\hline 3/29/08 & 8.06 & 1.4 & 13.28 & 148.31 & 48 \\
\hline 3/30/08 & 8.07 & 1.8 & 13.20 & 149.03 & 47 \\
\hline 3/31/08 & 8.06 & 2.3 & 13.12 & 149.04 & 45 \\
\hline 4/1/08 & 8.05 & 2.3 & 13.12 & 150.37 & 44 \\
\hline $4 / 2 / 08$ & 8.04 & 3.4 & 12.74 & 147.90 & 43 \\
\hline $4 / 3 / 08$ & 8.05 & 4.1 & 12.57 & 147.41 & 44 \\
\hline $4 / 4 / 08$ & 8.03 & 4.3 & 12.36 & 146.37 & 47 \\
\hline $4 / 5 / 08$ & 8.06 & 4.5 & 12.27 & 148.50 & 47 \\
\hline $4 / 6 / 08$ & 8.09 & 5.1 & 12.12 & 148.88 & 50 \\
\hline $4 / 7 / 08$ & 8.10 & 4.4 & 12.36 & 151.12 & 52 \\
\hline $4 / 8 / 08$ & 8.10 & 4.2 & 12.46 & 148.10 & 53 \\
\hline $4 / 9 / 08$ & 8.04 & 4.4 & 12.34 & 147.31 & 52 \\
\hline 4/10/08 & 8.01 & 5.2 & 12.21 & 143.87 & 54 \\
\hline 4/11/08 & 8.02 & 6.2 & 12.03 & 141.50 & 57 \\
\hline $4 / 12 / 08$ & 7.98 & 7.0 & 11.75 & 136.51 & 68 \\
\hline $4 / 13 / 08$ & 7.94 & 7.3 & 11.54 & 127.08 & 87 \\
\hline $4 / 14 / 08$ & 7.87 & 6.6 & 11.63 & 116.98 & 112 \\
\hline 4/15/08 & 7.82 & 4.6 & 12.27 & --- & 109 \\
\hline 4/16/08 & 7.85 & 5.1 & 12.23 & --- & 101 \\
\hline $4 / 17 / 08$ & 7.88 & 6.4 & 11.85 & --- & 100 \\
\hline 4/18/08 & 7.87 & 5.4 & 12.04 & 122.07 & 101 \\
\hline 4/19/08 & 7.87 & 3.5 & 12.54 & 124.40 & 96 \\
\hline 4/20/08 & 7.90 & 3.3 & 12.69 & 124.79 & 93 \\
\hline $4 / 21 / 08$ & 7.98 & 3.3 & 12.72 & 126.05 & 85 \\
\hline $4 / 22 / 08$ & 8.03 & 3.6 & 12.64 & 126.00 & 80 \\
\hline $4 / 23 / 08$ & 8.04 & 5.1 & 12.11 & 125.39 & 79 \\
\hline $4 / 24 / 08$ & 8.08 & 5.1 & 12.25 & 126.92 & 77 \\
\hline $4 / 25 / 08$ & 8.09 & 5.8 & 12.08 & 127.90 & 75 \\
\hline
\end{tabular}




\begin{tabular}{|c|c|c|c|c|c|}
\hline $4 / 26 / 08$ & 8.06 & 6.1 & 12.05 & 127.66 & 75 \\
\hline $4 / 27 / 08$ & 8.09 & 7.5 & 11.58 & 124.63 & 80 \\
\hline 4/28/08 & 8.05 & 8.1 & 11.27 & 120.76 & 90 \\
\hline $4 / 29 / 08$ & 8.02 & 7.1 & 11.47 & 112.58 & 109 \\
\hline 4/30/08 & 8.03 & 5.7 & 11.95 & 111.37 & 108 \\
\hline 5/1/08 & 8.02 & 5.5 & 12.11 & 111.72 & 104 \\
\hline $5 / 2 / 08$ & 8.03 & 6.3 & 11.91 & 112.18 & 103 \\
\hline $5 / 3 / 08$ & 8.03 & 6.9 & 11.67 & 111.50 & 106 \\
\hline $5 / 4 / 08$ & 8.00 & 8.4 & 11.24 & 107.90 & 115 \\
\hline $5 / 5 / 08$ & 7.93 & 8.2 & 11.21 & 97.41 & 140 \\
\hline $5 / 6 / 08$ & 7.87 & 7.7 & 11.37 & 85.01 & 165 \\
\hline $5 / 7 / 08$ & 7.79 & 7.3 & 11.51 & 80.28 & 178 \\
\hline $5 / 8 / 08$ & 7.75 & 6.3 & 11.83 & 78.35 & 179 \\
\hline $5 / 9 / 08$ & 7.79 & 6.3 & 11.83 & 79.37 & 169 \\
\hline 5/10/08 & 7.80 & 6.7 & 11.66 & 79.75 & 163 \\
\hline $5 / 11 / 08$ & 7.81 & 6.7 & 11.67 & 78.25 & 168 \\
\hline $5 / 12 / 08$ & 7.78 & 6.6 & 11.74 & 78.43 & 163 \\
\hline $5 / 13 / 08$ & 7.77 & 6.8 & 11.68 & 76.73 & 169 \\
\hline $5 / 14 / 08$ & 7.73 & 8.6 & 11.30 & 74.74 & 179 \\
\hline $5 / 15 / 08$ & 7.55 & 8.6 & 11.31 & 65.75 & 255 \\
\hline $5 / 16 / 08$ & 7.03 & 7.9 & 11.47 & 55.27 & 325 \\
\hline $5 / 17 / 08$ & 7.11 & 7.5 & 11.56 & 47.40 & 460 \\
\hline 5/18/08 & 7.25 & 7.1 & 11.64 & 40.19 & 643 \\
\hline 5/19/08 & 7.23 & 6.5 & 11.83 & 38.68 & 729 \\
\hline $5 / 20 / 08$ & 7.23 & 6.0 & 11.97 & 39.77 & 730 \\
\hline $5 / 21 / 08$ & 7.28 & 5.8 & 12.04 & 40.86 & 676 \\
\hline $5 / 22 / 08$ & 7.34 & 6.1 & 11.87 & 42.94 & 591 \\
\hline $5 / 23 / 08$ & 7.37 & 5.9 & 11.90 & 44.61 & 537 \\
\hline $5 / 24 / 08$ & 7.33 & 6.7 & 11.70 & 43.58 & 542 \\
\hline $5 / 25 / 08$ & 7.38 & 7.0 & 11.68 & 42.67 & 564 \\
\hline $5 / 26 / 08$ & 7.31 & 6.9 & 11.70 & 41.60 & 595 \\
\hline $5 / 27 / 08$ & 7.29 & 7.1 & 11.68 & 43.08 & 583 \\
\hline $5 / 28 / 08$ & 7.33 & 6.9 & 11.70 & 43.18 & 567 \\
\hline $5 / 29 / 08$ & 7.31 & 7.3 & 11.58 & 42.67 & 576 \\
\hline $5 / 30 / 08$ & 7.34 & 6.7 & 11.79 & 42.13 & 570 \\
\hline $5 / 31 / 08$ & 7.36 & 6.9 & 11.73 & 41.73 & 539 \\
\hline 6/1/08 & 7.38 & 7.5 & 11.56 & 43.95 & 522 \\
\hline $6 / 2 / 08$ & 7.34 & 6.9 & 11.72 & 41.33 & 519 \\
\hline $6 / 3 / 08$ & 7.40 & 6.1 & 11.85 & 42.85 & 481 \\
\hline $6 / 4 / 08$ & 7.36 & 6.0 & 11.93 & 43.48 & 434 \\
\hline 6/5/08 & 7.40 & 6.1 & 11.87 & 44.43 & 394 \\
\hline 6/6/08 & 7.44 & 5.9 & 11.91 & 45.50 & 353 \\
\hline $6 / 7 / 08$ & 7.51 & 6.8 & 11.73 & 47.58 & 318 \\
\hline $6 / 8 / 08$ & 7.55 & 7.2 & 11.62 & 50.38 & 302 \\
\hline 6/9/08 & 7.54 & 7.2 & 11.49 & 50.76 & 290 \\
\hline 6/10/08 & 7.47 & 6.0 & 11.88 & 51.82 & 277 \\
\hline 6/11/08 & 7.69 & 7.4 & 11.54 & 52.38 & 261 \\
\hline 6/12/08 & 7.73 & 8.2 & 11.33 & 51.67 & 261 \\
\hline 6/13/08 & 7.68 & 9.0 & 11.10 & 49.59 & 273 \\
\hline 6/14/08 & 7.63 & 8.2 & 11.35 & 47.67 & 285 \\
\hline 6/15/08 & 7.60 & 8.4 & 11.26 & 46.28 & 292 \\
\hline
\end{tabular}




\begin{tabular}{|c|c|c|c|c|c|}
\hline 6/16/08 & 7.58 & 8.7 & 11.17 & 45.08 & 296 \\
\hline 6/17/08 & 7.55 & 8.9 & 11.16 & 44.31 & 298 \\
\hline 6/18/08 & 7.53 & 8.1 & 11.40 & 44.74 & 290 \\
\hline 6/19/08 & 7.56 & 8.2 & 11.37 & 45.54 & 276 \\
\hline 6/20/08 & 7.58 & 9.2 & 11.08 & 46.00 & 265 \\
\hline 6/21/08 & 7.51 & 8.9 & 11.11 & 45.60 & 268 \\
\hline $6 / 22 / 08$ & 7.53 & 9.2 & 11.08 & 44.94 & 267 \\
\hline 6/23/08 & 7.50 & 8.4 & 11.28 & 44.86 & 262 \\
\hline 6/24/08 & 7.52 & 9.3 & 11.04 & 45.88 & 243 \\
\hline $6 / 25 / 08$ & 7.54 & 9.5 & 10.96 & 46.92 & 231 \\
\hline 6/26/08 & 7.53 & 10.2 & 10.78 & 47.63 & 223 \\
\hline $6 / 27 / 08$ & 7.47 & 10.8 & 10.65 & 48.09 & 219 \\
\hline 6/28/08 & 7.46 & 12.0 & 10.33 & 48.59 & 218 \\
\hline 6/29/08 & 7.42 & 12.7 & 10.13 & 47.74 & 220 \\
\hline 6/30/08 & 7.38 & 13.6 & 9.89 & 47.43 & 219 \\
\hline $7 / 1 / 08$ & 7.38 & 13.5 & 9.92 & 48.93 & 212 \\
\hline $7 / 2 / 08$ & 7.43 & 13.7 & 9.88 & 50.52 & 196 \\
\hline $7 / 3 / 08$ & 7.44 & 14.5 & 9.66 & 52.63 & 180 \\
\hline $7 / 4 / 08$ & 7.54 & 14.1 & 9.74 & 54.83 & 167 \\
\hline $7 / 5 / 08$ & 7.54 & 13.0 & 10.03 & 57.17 & 156 \\
\hline $7 / 6 / 08$ & 7.55 & 13.0 & 10.03 & 59.21 & 147 \\
\hline $7 / 7 / 08$ & 7.65 & 12.8 & 10.09 & 61.52 & 138 \\
\hline $7 / 8 / 08$ & 7.64 & 13.7 & 9.93 & 64.48 & 133 \\
\hline $7 / 9 / 08$ & 7.65 & 14.6 & 9.67 & 67.16 & 128 \\
\hline 7/10/08 & 7.69 & 13.9 & 9.83 & 69.11 & 123 \\
\hline 7/11/08 & 7.68 & 12.1 & 10.33 & 71.11 & 118 \\
\hline $7 / 12 / 08$ & 7.71 & 12.9 & 10.14 & 72.41 & 114 \\
\hline $7 / 13 / 08$ & 7.74 & 14.1 & 9.80 & 73.68 & 110 \\
\hline $7 / 14 / 08$ & 7.77 & 14.5 & 9.69 & 75.08 & 107 \\
\hline $7 / 15 / 08$ & 7.76 & 14.6 & 9.67 & 76.19 & 105 \\
\hline 7/16/08 & 7.75 & 14.6 & 9.69 & 77.10 & 104 \\
\hline 7/17/08 & 7.79 & 14.6 & 9.69 & 78.31 & 102 \\
\hline 7/18/08 & 7.80 & 14.6 & 9.67 & 79.43 & 100 \\
\hline 7/19/08 & 7.79 & 14.4 & 9.70 & 80.64 & 99 \\
\hline $7 / 20 / 08$ & 7.79 & 15.0 & 9.57 & 84.29 & 96 \\
\hline $7 / 21 / 08$ & 7.82 & 15.6 & 9.43 & 85.97 & 93 \\
\hline $7 / 22 / 08$ & 7.83 & 16.1 & 9.33 & 89.49 & 92 \\
\hline $7 / 23 / 08$ & 7.85 & 14.6 & 9.69 & 88.74 & 85 \\
\hline $7 / 24 / 08$ & 7.82 & 14.5 & 9.74 & 89.20 & 75 \\
\hline $7 / 25 / 08$ & 7.87 & 14.7 & 9.66 & 91.99 & 73 \\
\hline $7 / 26 / 08$ & 7.88 & 15.3 & 9.51 & 93.60 & 70 \\
\hline $7 / 27 / 08$ & 7.90 & 15.4 & 9.47 & 94.57 & 69 \\
\hline $7 / 28 / 08$ & 7.88 & 14.7 & 9.64 & 95.42 & 68 \\
\hline $7 / 29 / 08$ & 7.97 & 14.1 & 9.80 & 96.40 & 66 \\
\hline 7/30/08 & 8.00 & 13.7 & 9.90 & 96.85 & 66 \\
\hline 7/31/08 & 7.98 & 13.1 & 9.98 & 96.33 & 64 \\
\hline 8/1/08 & 7.97 & 14.6 & 9.66 & 98.13 & 63 \\
\hline $8 / 2 / 08$ & 7.95 & 13.6 & 9.90 & 99.06 & 62 \\
\hline 8/3/08 & 7.96 & 13.7 & 9.89 & 99.65 & 61 \\
\hline $8 / 4 / 08$ & 7.96 & 14.2 & 9.80 & 100.20 & 58 \\
\hline 8/5/08 & 7.95 & 15.0 & 9.63 & 100.96 & 50 \\
\hline
\end{tabular}




\begin{tabular}{|c|c|c|c|c|c|}
\hline 8/6/08 & 7.94 & 15.1 & 9.60 & 101.80 & 42 \\
\hline 8/7/08 & 7.97 & 15.7 & 9.46 & 102.44 & 41 \\
\hline 8/8/08 & 8.03 & 16.0 & 9.36 & 103.08 & 40 \\
\hline 8/9/08 & 7.99 & 15.8 & 9.40 & 103.80 & 41 \\
\hline 8/10/08 & 8.04 & 14.2 & 9.82 & 103.76 & 41 \\
\hline 8/11/08 & 8.00 & 13.7 & 9.92 & 104.53 & 40 \\
\hline 8/12/08 & 8.03 & 14.5 & 9.74 & 104.94 & 39 \\
\hline 8/13/08 & 8.02 & 15.8 & 9.44 & 105.42 & 38 \\
\hline 8/14/08 & 8.01 & 16.6 & 9.30 & 106.20 & 37 \\
\hline 8/15/08 & 8.00 & 17.0 & 9.21 & 107.05 & 35 \\
\hline 8/16/08 & 8.01 & 17.7 & 9.03 & 107.82 & 34 \\
\hline 8/17/08 & 8.04 & 18.3 & 8.91 & 108.25 & 32 \\
\hline 8/18/08 & 8.01 & 18.5 & 8.83 & 108.95 & 31 \\
\hline 8/19/08 & 8.07 & 17.2 & 9.08 & 109.70 & 32 \\
\hline 8/20/08 & 8.09 & 16.1 & 9.28 & 109.16 & 35 \\
\hline 8/21/08 & 8.07 & 14.2 & 9.71 & 105.40 & 39 \\
\hline 8/22/08 & 8.07 & 12.4 & 10.22 & 106.12 & 37 \\
\hline 8/23/08 & 8.09 & 13.3 & 9.99 & 107.62 & 34 \\
\hline 8/24/08 & 8.04 & 14.1 & 9.76 & 108.79 & 31 \\
\hline 8/25/08 & 8.07 & 14.3 & 9.72 & 109.13 & 33 \\
\hline 8/26/08 & 8.11 & 12.6 & 10.14 & 109.57 & 31 \\
\hline 8/27/08 & 8.09 & 12.6 & 10.13 & 110.44 & 30 \\
\hline 8/28/08 & 8.09 & 12.9 & 10.13 & 110.67 & 31 \\
\hline 8/29/08 & 8.07 & 14.3 & 9.70 & 110.57 & 31 \\
\hline 8/30/08 & 8.10 & 13.3 & 9.90 & 111.42 & 29 \\
\hline 8/31/08 & 8.10 & 11.2 & 10.42 & 112.59 & 28 \\
\hline 9/1/08 & 8.09 & 10.7 & 10.61 & 111.95 & 29 \\
\hline 9/2/08 & 8.10 & 10.8 & 10.64 & 112.14 & 28 \\
\hline 9/3/08 & 8.09 & 11.5 & 10.41 & 112.63 & 27 \\
\hline 9/4/08 & 8.09 & 12.3 & 10.25 & 113.05 & 26 \\
\hline 9/5/08 & 8.13 & 12.9 & 10.10 & 113.49 & 25 \\
\hline 9/6/08 & 8.08 & 12.7 & 10.15 & 114.23 & 25 \\
\hline 9/7/08 & 8.11 & 12.8 & 10.12 & 114.79 & 25 \\
\hline 9/8/08 & 8.12 & 11.9 & 10.29 & 115.86 & 24 \\
\hline 9/9/08 & 8.12 & 12.4 & 10.10 & 116.14 & 24 \\
\hline 9/10/08 & 8.10 & 11.5 & 10.39 & 116.99 & 23 \\
\hline 9/11/08 & 8.10 & 11.5 & 10.40 & 117.40 & 23 \\
\hline 9/12/08 & 8.13 & 12.2 & 10.20 & 117.38 & 23 \\
\hline 9/13/08 & 8.11 & 12.0 & 10.31 & 118.13 & 22 \\
\hline 9/14/08 & 8.11 & 11.1 & 10.55 & 118.74 & 22 \\
\hline 9/15/08 & 8.12 & 11.0 & 10.57 & 119.08 & 22 \\
\hline 9/16/08 & 8.14 & 11.4 & 10.49 & 119.28 & 21 \\
\hline 9/17/08 & 8.15 & 11.9 & 10.34 & 119.42 & 20 \\
\hline 9/18/08 & 8.14 & 12.3 & 10.23 & 119.88 & 20 \\
\hline 9/19/08 & 8.15 & 12.6 & 10.15 & 120.27 & 20 \\
\hline 9/20/08 & 8.14 & 13.0 & 10.00 & 120.84 & 20 \\
\hline 9/21/08 & 8.17 & 12.1 & 10.27 & 120.49 & 22 \\
\hline 9/22/08 & 8.15 & 10.4 & 10.67 & 120.21 & 22 \\
\hline 9/23/08 & 8.17 & 8.0 & 11.32 & 122.09 & 22 \\
\hline 9/24/08 & 8.16 & 8.7 & 11.07 & 121.74 & 22 \\
\hline 9/25/08 & 8.16 & 9.1 & 10.96 & 121.48 & 22 \\
\hline
\end{tabular}




\begin{tabular}{|c|c|c|c|c|c|}
\hline 9/26/08 & 8.17 & 9.0 & 11.07 & 121.76 & 22 \\
\hline 9/27/08 & 8.17 & 9.2 & 10.98 & 121.87 & 22 \\
\hline 9/28/08 & 8.16 & 8.6 & 11.16 & 122.55 & 22 \\
\hline 9/29/08 & 8.15 & 8.6 & 11.16 & 122.94 & 22 \\
\hline 9/30/08 & 8.18 & 9.0 & 11.06 & 123.20 & 22 \\
\hline 10/1/08 & 8.17 & 9.8 & 10.79 & 123.16 & 21 \\
\hline $10 / 2 / 08$ & 8.16 & 10.7 & 10.47 & 123.59 & 22 \\
\hline 10/3/08 & 8.15 & 10.1 & 10.56 & 124.14 & 22 \\
\hline 10/4/08 & 8.07 & 10.8 & 10.29 & 115.24 & 41 \\
\hline 10/5/08 & 8.12 & 9.3 & 10.85 & 112.31 & 33 \\
\hline 10/6/08 & 8.15 & 8.8 & 11.02 & 118.40 & 27 \\
\hline 10/7/08 & 8.13 & 8.4 & 11.12 & 117.82 & 29 \\
\hline 10/8/08 & 7.99 & 6.6 & 11.70 & 116.23 & --- \\
\hline 10/9/08 & 8.02 & 5.7 & 11.90 & 120.75 & --- \\
\hline $10 / 10 / 08$ & 8.03 & 4.9 & 12.15 & 122.27 & --- \\
\hline $10 / 11 / 08$ & 8.03 & 3.9 & 12.47 & 123.11 & --- \\
\hline 10/12/08 & 8.02 & 5.3 & 12.07 & 124.07 & --- \\
\hline $10 / 13 / 08$ & 8.01 & 6.2 & 11.81 & 122.30 & --- \\
\hline $10 / 14 / 08$ & 8.01 & 7.0 & 11.54 & 119.32 & --- \\
\hline $10 / 15 / 08$ & 7.99 & 4.8 & 12.16 & 117.48 & --- \\
\hline 10/16/08 & 8.00 & 6.4 & 11.73 & 121.30 & --- \\
\hline $10 / 17 / 08$ & 7.99 & 7.5 & 11.33 & 120.59 & --- \\
\hline 10/18/08 & 8.00 & 8.1 & 11.08 & 120.18 & --- \\
\hline $10 / 19 / 08$ & 8.01 & 5.6 & 11.84 & 122.93 & --- \\
\hline $10 / 20 / 08$ & 8.01 & 5.9 & 11.69 & 123.94 & --- \\
\hline 10/21/08 & 7.98 & 5.3 & 12.01 & 122.09 & --- \\
\hline $10 / 22 / 08$ & 7.94 & 4.2 & 12.37 & 123.94 & --- \\
\hline $10 / 23 / 08$ & 7.95 & 3.7 & 12.39 & 122.63 & --- \\
\hline $10 / 24 / 08$ & 7.95 & 4.4 & 12.21 & 122.75 & --- \\
\hline $10 / 25 / 08$ & 7.95 & 5.4 & 11.89 & 123.77 & --- \\
\hline $10 / 26 / 08$ & 7.94 & 3.8 & 12.48 & 124.96 & --- \\
\hline $10 / 27 / 08$ & 7.95 & 2.8 & 12.72 & 125.52 & --- \\
\hline $10 / 28 / 08$ & 7.94 & 3.0 & 12.67 & 125.14 & --- \\
\hline 10/29/08 & 7.94 & 3.1 & 12.51 & 124.41 & --- \\
\hline $10 / 30 / 08$ & 7.92 & 4.0 & 12.22 & 125.42 & --- \\
\hline $10 / 31 / 08$ & 7.90 & 5.7 & 11.65 & 124.18 & --- \\
\hline 11/1/08 & 7.87 & 5.8 & 11.58 & 121.38 & --- \\
\hline 11/2/08 & 7.86 & 6.4 & 11.28 & 118.27 & --- \\
\hline 11/3/08 & 7.87 & 5.6 & 11.57 & 115.30 & --- \\
\hline $11 / 4 / 08$ & 7.87 & 5.4 & 11.54 & 110.60 & --- \\
\hline $11 / 5 / 08$ & 7.90 & 2.9 & 12.54 & 117.04 & --- \\
\hline 11/6/08 & 7.91 & 2.9 & 12.65 & 118.04 & --- \\
\hline 11/7/08 & 7.88 & 3.4 & 12.50 & 102.97 & --- \\
\hline 11/8/08 & 7.67 & 4.0 & 12.19 & 80.26 & --- \\
\hline 11/9/08 & 7.71 & 4.4 & 12.05 & 90.63 & --- \\
\hline 11/10/08 & 7.79 & 4.0 & 12.19 & 97.85 & --- \\
\hline 11/11/08 & 7.80 & 4.4 & 12.08 & 100.76 & --- \\
\hline 11/12/08 & 7.66 & 5.1 & 11.89 & 77.35 & --- \\
\hline 11/13/08 & 7.77 & 4.3 & 12.32 & 77.53 & --- \\
\hline $11 / 14 / 08$ & 7.77 & 3.2 & 12.75 & 84.38 & --- \\
\hline 11/15/08 & 7.75 & 3.2 & 12.71 & 82.60 & --- \\
\hline
\end{tabular}




$\begin{array}{cccccc}11 / 16 / 08 & 7.72 & 2.8 & 12.78 & 81.16 & --- \\ 11 / 17 / 08 & 7.73 & 2.8 & 12.79 & 81.23 & --- \\ 11 / 18 / 08 & 7.73 & 3.2 & 12.63 & 80.89 & --- \\ 11 / 19 / 08 & 7.73 & 2.9 & 12.80 & 81.36 & -- \\ 11 / 20 / 08 & 7.74 & 3.2 & 12.58 & 81.59 & -- \\ 11 / 21 / 08 & 7.74 & 3.0 & 12.70 & 81.92 & -- \\ 11 / 22 / 08 & 7.77 & 3.0 & 12.68 & 82.38 & -- \\ 11 / 23 / 08 & 7.78 & 1.3 & 13.25 & 86.13 & -- \\ 11 / 24 / 08 & 7.78 & 0.7 & 13.41 & 88.07 & -- \\ 11 / 25 / 08 & 7.77 & 1.1 & 13.28 & 85.44 & -- \\ 11 / 26 / 08 & 7.78 & 0.4 & 13.51 & 86.67 & -- \\ 11 / 27 / 08 & 7.79 & -0.1 & 13.61 & 92.01 & -- \\ 11 / 28 / 08 & 7.77 & 0.9 & 13.44 & 86.26 & -- \\ 11 / 29 / 08 & 7.76 & 1.7 & 13.17 & 86.46 & -- \\ 11 / 30 / 08 & 7.76 & 2.3 & 12.98 & 85.67 & -- \\ 12 / 1 / 08 & 7.78 & 2.5 & 12.77 & 87.19 & -- \\ 12 / 2 / 08 & 7.76 & 2.8 & 12.73 & 86.97 & -- \\ 12 / 3 / 08 & --- & --- & -- & -\end{array}$


Appendix C. Daily medians $(\mathrm{pH})$ or means of water quality parameters at the Ardenvoir gage. Discharge is from the Ardenvoir gage.

\begin{tabular}{|c|c|c|c|c|c|}
\hline Date & $\mathrm{pH}$ & Temperature (C) & $\begin{array}{c}\text { Dissolved } \\
\text { Oxygen } \\
(\mathrm{mg} / \mathrm{L})\end{array}$ & $\begin{array}{c}\text { Specific } \\
\text { Conductivity } \\
(\mathrm{mS} / \mathrm{cm})\end{array}$ & Discharge (cfs) \\
\hline 3/13/08 & & & & & 106 \\
\hline 3/14/08 & 7.42 & 4.0 & 11.54 & 57.32 & 106 \\
\hline $3 / 15 / 08$ & 7.45 & 4.4 & 11.48 & 56.65 & 107 \\
\hline $3 / 16 / 08$ & 7.45 & 3.7 & 11.75 & 56.96 & 105 \\
\hline 3/17/08 & 7.46 & 4.3 & 11.63 & 56.90 & 105 \\
\hline 3/18/08 & 7.46 & 4.2 & 11.62 & 56.81 & 105 \\
\hline 3/19/08 & 7.46 & 3.8 & 11.75 & 56.93 & 104 \\
\hline 3/20/08 & 7.46 & 3.8 & 11.72 & 56.97 & 103 \\
\hline 3/21/08 & 7.47 & 4.0 & 11.81 & 56.88 & 100 \\
\hline 3/22/08 & 7.47 & 3.8 & 11.99 & 57.11 & 98 \\
\hline 3/23/08 & 7.48 & 3.8 & 11.78 & 56.96 & 100 \\
\hline 3/24/08 & 7.53 & 3.6 & 11.88 & 56.47 & 99 \\
\hline $3 / 25 / 08$ & 7.54 & 3.7 & 11.86 & 56.98 & 96 \\
\hline $3 / 26 / 08$ & 7.54 & 4.1 & 11.65 & 56.91 & 97 \\
\hline 3/27/08 & 7.54 & 4.2 & 11.73 & 56.84 & 94 \\
\hline 3/28/08 & 7.54 & 2.0 & 12.06 & 57.08 & 93 \\
\hline 3/29/08 & 7.54 & 2.0 & 12.24 & 57.26 & 91 \\
\hline 3/30/08 & 7.54 & 2.7 & 12.09 & 56.94 & 90 \\
\hline 3/31/08 & 7.56 & 2.9 & 12.14 & 57.56 & 87 \\
\hline 4/1/08 & 7.56 & 3.7 & 11.99 & 57.69 & 85 \\
\hline $4 / 2 / 08$ & 7.57 & 4.8 & 11.64 & 56.85 & 89 \\
\hline 4/3/08 & 7.57 & 4.9 & 11.62 & 57.10 & 88 \\
\hline 4/4/08 & 7.58 & 4.8 & 11.48 & 57.04 & 88 \\
\hline 4/5/08 & 7.57 & 5.1 & 11.47 & 57.23 & 88 \\
\hline 4/6/08 & 7.59 & 6.1 & 11.18 & 56.95 & 89 \\
\hline 4/7/08 & 7.58 & 5.2 & 11.40 & 56.81 & 89 \\
\hline 4/8/08 & 7.60 & 4.6 & 11.62 & 57.20 & 88 \\
\hline 4/9/08 & 7.52 & 5.2 & 11.44 & 57.44 & 87 \\
\hline 4/10/08 & 7.37 & 6.2 & 11.27 & 57.54 & 88 \\
\hline 4/11/08 & 7.37 & 6.8 & 11.26 & 57.14 & 89 \\
\hline 4/12/08 & 7.35 & 7.7 & 11.01 & 56.60 & 94 \\
\hline 4/13/08 & 7.34 & 8.2 & 10.79 & 54.43 & 113 \\
\hline 4/14/08 & 7.31 & 7.9 & 10.73 & 52.37 & 140 \\
\hline 4/15/08 & 7.27 & 5.5 & 11.35 & 51.90 & 148 \\
\hline 4/16/08 & 7.24 & 5.7 & 11.49 & 52.20 & 145 \\
\hline $4 / 17 / 08$ & 7.24 & 6.8 & 11.17 & 51.47 & 152 \\
\hline 4/18/08 & 7.25 & 5.8 & 11.30 & 50.57 & 164 \\
\hline 4/19/08 & 7.23 & 4.4 & 11.61 & 50.44 & 167 \\
\hline 4/20/08 & 7.25 & 3.3 & 11.93 & 49.76 & 171 \\
\hline 4/21/08 & 7.29 & 3.6 & 12.03 & 50.42 & 166 \\
\hline $4 / 22 / 08$ & 7.31 & 3.7 & 11.95 & 50.62 & 162 \\
\hline 4/23/08 & 7.32 & 5.4 & 11.47 & 50.40 & 162 \\
\hline 4/24/08 & 7.33 & 5.0 & 11.56 & 50.62 & 157 \\
\hline
\end{tabular}




\begin{tabular}{|c|c|c|c|c|c|}
\hline $4 / 25 / 08$ & 7.34 & 6.2 & 11.40 & 51.08 & 153 \\
\hline $4 / 26 / 08$ & 7.34 & 6.9 & 11.32 & 51.17 & 152 \\
\hline $4 / 27 / 08$ & 7.35 & 7.7 & 11.07 & 50.80 & 157 \\
\hline $4 / 28 / 08$ & 7.37 & 8.4 & 10.77 & 50.33 & 168 \\
\hline 4/29/08 & 7.38 & 7.8 & 10.82 & 49.32 & 193 \\
\hline 4/30/08 & 7.36 & 6.4 & 11.22 & 49.18 & 198 \\
\hline $5 / 1 / 08$ & 7.34 & 6.4 & 11.36 & 48.96 & 202 \\
\hline $5 / 2 / 08$ & 7.34 & 6.5 & 11.35 & 48.47 & 212 \\
\hline $5 / 3 / 08$ & 7.34 & 6.7 & 11.22 & 47.94 & 223 \\
\hline $5 / 4 / 08$ & 7.32 & 8.1 & 10.94 & 47.26 & 241 \\
\hline $5 / 5 / 08$ & 7.30 & 7.9 & 10.92 & 44.72 & 306 \\
\hline $5 / 6 / 08$ & 7.26 & 7.1 & 11.19 & 42.05 & 390 \\
\hline $5 / 7 / 08$ & 7.39 & 6.9 & 11.30 & 40.09 & 468 \\
\hline $5 / 8 / 08$ & 7.23 & 5.6 & 11.65 & 39.21 & 521 \\
\hline $5 / 9 / 08$ & 6.88 & 6.1 & 11.53 & 39.02 & 515 \\
\hline 5/10/08 & 6.87 & 6.0 & 11.48 & 39.20 & 510 \\
\hline 5/11/08 & 6.87 & 6.6 & 11.35 & 39.21 & 512 \\
\hline $5 / 12 / 08$ & 6.88 & 6.5 & 11.44 & 39.34 & 502 \\
\hline $5 / 13 / 08$ & 6.90 & 6.4 & 11.39 & 38.98 & 529 \\
\hline $5 / 14 / 08$ & 6.89 & 7.5 & 11.30 & 38.31 & 577 \\
\hline $5 / 15 / 08$ & 6.92 & 7.1 & 11.52 & --- & 877 \\
\hline $5 / 16 / 08$ & 6.79 & 6.3 & 11.70 & --- & 1355 \\
\hline $5 / 17 / 08$ & 6.70 & 6.1 & 11.69 & --- & 2006 \\
\hline 5/18/08 & 6.62 & 5.9 & 11.56 & --- & 2924 \\
\hline 5/19/08 & 6.83 & 5.9 & 11.48 & --- & 3010 \\
\hline $5 / 20 / 08$ & 6.84 & 5.7 & 11.49 & 30.52 & 2823 \\
\hline $5 / 21 / 08$ & 6.84 & 5.8 & 11.55 & 31.40 & 2446 \\
\hline $5 / 22 / 08$ & 6.89 & 5.8 & 11.52 & 32.83 & 1924 \\
\hline $5 / 23 / 08$ & 6.94 & 5.7 & 11.46 & 33.48 & 1648 \\
\hline $5 / 24 / 08$ & 6.98 & 6.4 & 11.41 & 33.11 & 1612 \\
\hline $5 / 25 / 08$ & 6.92 & 6.6 & 11.42 & 32.21 & 1704 \\
\hline $5 / 26 / 08$ & 6.95 & 6.6 & 11.40 & 31.52 & 1866 \\
\hline $5 / 27 / 08$ & 6.97 & 6.8 & 11.41 & 31.43 & 1938 \\
\hline $5 / 28 / 08$ & 6.99 & 6.5 & 11.43 & 31.26 & 1992 \\
\hline $5 / 29 / 08$ & 7.01 & 7.0 & 11.32 & 31.10 & 1970 \\
\hline 5/30/08 & 7.04 & 6.6 & 11.45 & 30.85 & 1994 \\
\hline 5/31/08 & 7.08 & 6.8 & 11.43 & 31.04 & 1908 \\
\hline $6 / 1 / 08$ & 7.13 & 7.2 & 11.30 & 31.00 & 1838 \\
\hline $6 / 2 / 08$ & 7.09 & 6.6 & 11.44 & 30.96 & 1813 \\
\hline $6 / 3 / 08$ & 7.13 & 5.9 & 11.50 & 31.77 & 1631 \\
\hline $6 / 4 / 08$ & 7.10 & 6.1 & 11.46 & 32.65 & 1424 \\
\hline $6 / 5 / 08$ & 7.15 & 6.1 & 11.44 & 33.39 & 1258 \\
\hline $6 / 6 / 08$ & 7.17 & 6.3 & 11.40 & 34.04 & 1121 \\
\hline $6 / 7 / 08$ & 7.19 & 7.0 & 11.32 & 34.70 & 1003 \\
\hline $6 / 8 / 08$ & 7.18 & 7.2 & 11.29 & 34.97 & 936 \\
\hline $6 / 9 / 08$ & 7.23 & 7.0 & 11.24 & 35.04 & 900 \\
\hline 6/10/08 & 7.20 & 6.4 & 11.39 & 35.23 & 868 \\
\hline 6/11/08 & 7.14 & 7.7 & 11.15 & 35.59 & 820 \\
\hline 6/12/08 & 7.12 & 8.3 & 11.03 & 35.25 & 820 \\
\hline 6/13/08 & 7.10 & 8.9 & 10.86 & 33.99 & 934 \\
\hline 6/14/08 & 7.08 & 7.7 & 11.22 & 32.71 & 1091 \\
\hline
\end{tabular}




\begin{tabular}{|c|c|c|c|c|c|}
\hline 6/15/08 & 7.07 & 7.9 & 11.14 & 32.29 & 1126 \\
\hline 6/16/08 & 7.07 & 8.1 & 11.07 & 31.81 & 1160 \\
\hline 6/17/08 & 7.08 & 8.1 & 11.10 & 31.78 & 1175 \\
\hline 6/18/08 & 7.09 & 7.5 & 11.28 & 31.76 & 1140 \\
\hline 6/19/08 & 7.10 & 7.4 & 11.30 & 32.51 & 1061 \\
\hline 6/20/08 & 7.12 & 8.3 & 11.05 & 32.93 & 990 \\
\hline 6/21/08 & 7.09 & 8.0 & 11.02 & 32.82 & 1021 \\
\hline 6/22/08 & 7.13 & 8.6 & 10.98 & 32.14 & 1058 \\
\hline 6/23/08 & 7.13 & 7.7 & 11.18 & 31.85 & 1082 \\
\hline 6/24/08 & 7.13 & 8.5 & 11.01 & 32.32 & 1008 \\
\hline $6 / 25 / 08$ & 7.14 & 8.5 & 10.96 & 32.63 & 964 \\
\hline 6/26/08 & 7.13 & 9.2 & 10.81 & 32.98 & 942 \\
\hline $6 / 27 / 08$ & 7.09 & 9.8 & 10.68 & 32.89 & 974 \\
\hline 6/28/08 & 7.04 & 10.5 & 10.51 & 31.51 & 1112 \\
\hline 6/29/08 & 7.03 & 10.6 & 10.43 & 30.34 & 1256 \\
\hline 6/30/08 & 7.07 & 10.8 & 10.33 & 29.83 & 1358 \\
\hline 7/1/08 & 7.06 & 10.7 & 10.38 & 29.73 & 1360 \\
\hline $7 / 2 / 08$ & 7.05 & 11.0 & 10.32 & 30.11 & 1266 \\
\hline $7 / 3 / 08$ & 7.09 & 11.2 & 10.20 & 30.61 & 1169 \\
\hline $7 / 4 / 08$ & 7.09 & 10.9 & 10.26 & 30.99 & 1089 \\
\hline $7 / 5 / 08$ & 7.15 & 10.2 & 10.46 & 32.44 & 925 \\
\hline 7/6/08 & 7.17 & 10.8 & 10.31 & 33.68 & 787 \\
\hline $7 / 7 / 08$ & 7.15 & 11.1 & 10.25 & 34.00 & 703 \\
\hline $7 / 8 / 08$ & 7.14 & 11.7 & 10.13 & 34.53 & 646 \\
\hline $7 / 9 / 08$ & 7.14 & 12.4 & 9.94 & 35.52 & 623 \\
\hline $7 / 10 / 08$ & 7.18 & 12.0 & 9.99 & 35.46 & 609 \\
\hline 7/11/08 & 7.20 & 10.5 & 10.45 & 36.45 & 548 \\
\hline $7 / 12 / 08$ & 7.22 & 11.5 & 10.18 & 37.51 & 490 \\
\hline 7/13/08 & 7.25 & 12.5 & 9.89 & 38.03 & 459 \\
\hline 7/14/08 & 7.25 & 12.7 & 9.81 & 37.99 & 450 \\
\hline 7/15/08 & 7.28 & 12.7 & 9.80 & 38.12 & 429 \\
\hline $7 / 16 / 08$ & 7.39 & 13.0 & 9.78 & 38.35 & 405 \\
\hline $7 / 17 / 08$ & 7.42 & 13.0 & 9.77 & 38.94 & 388 \\
\hline 7/18/08 & 7.44 & 13.0 & 9.74 & 39.64 & 367 \\
\hline 7/19/08 & 7.42 & 13.1 & 9.71 & 40.44 & 348 \\
\hline $7 / 20 / 08$ & 7.44 & 13.8 & 9.56 & 41.11 & 333 \\
\hline $7 / 21 / 08$ & 7.43 & 14.1 & 9.47 & 41.40 & 323 \\
\hline $7 / 22 / 08$ & 7.46 & 14.6 & 9.35 & 41.47 & 320 \\
\hline $7 / 23 / 08$ & 7.45 & 14.0 & 9.53 & 40.86 & 316 \\
\hline $7 / 24 / 08$ & 7.42 & 13.9 & 9.58 & 41.02 & 300 \\
\hline $7 / 25 / 08$ & 7.45 & 13.8 & 9.57 & 41.78 & 283 \\
\hline $7 / 26 / 08$ & 7.45 & 14.1 & 9.43 & 42.24 & 275 \\
\hline $7 / 27 / 08$ & 7.44 & 14.4 & 9.38 & 42.35 & 270 \\
\hline $7 / 28 / 08$ & 7.43 & 14.2 & 9.45 & 42.47 & 262 \\
\hline $7 / 29 / 08$ & 7.45 & 13.1 & 9.62 & 42.89 & 250 \\
\hline 7/30/08 & 7.44 & 13.1 & 9.66 & 43.28 & 242 \\
\hline 7/31/08 & 7.41 & 13.2 & 9.62 & 44.27 & 226 \\
\hline 8/1/08 & 7.44 & 14.1 & 9.43 & 44.94 & 206 \\
\hline $8 / 2 / 08$ & 7.44 & 13.1 & 9.65 & 45.22 & 198 \\
\hline 8/3/08 & 7.42 & 13.4 & 9.60 & 45.47 & 187 \\
\hline 8/4/08 & 7.43 & 14.3 & 9.42 & 46.32 & 175 \\
\hline
\end{tabular}




\begin{tabular}{|c|c|c|c|c|c|}
\hline $8 / 5 / 08$ & 7.43 & 15.0 & 9.25 & 47.02 & 169 \\
\hline 8/6/08 & 7.44 & 15.0 & 9.20 & 47.13 & 167 \\
\hline $8 / 7 / 08$ & 7.45 & 15.5 & 9.11 & 46.79 & 169 \\
\hline 8/8/08 & 7.45 & 15.4 & 9.08 & 46.25 & 173 \\
\hline 8/9/08 & 7.43 & 15.5 & 9.04 & 45.47 & 185 \\
\hline 8/10/08 & 7.47 & 14.4 & 9.35 & 45.48 & 173 \\
\hline 8/11/08 & 7.43 & 13.7 & 9.47 & 46.56 & 160 \\
\hline 8/12/08 & 7.40 & 14.2 & 9.41 & 47.61 & 150 \\
\hline 8/13/08 & 7.45 & 15.4 & 9.17 & 48.24 & 145 \\
\hline 8/14/08 & 7.45 & 16.4 & 8.96 & 48.44 & 143 \\
\hline 8/15/08 & 7.48 & 17.0 & 8.83 & 48.32 & 144 \\
\hline 8/16/08 & 7.47 & 17.6 & 8.68 & 47.67 & 148 \\
\hline 8/17/08 & 7.46 & 17.7 & 8.62 & 46.85 & 152 \\
\hline 8/18/08 & 7.47 & 17.0 & 8.66 & 46.33 & 152 \\
\hline 8/19/08 & 7.45 & 16.3 & 8.84 & 46.29 & 152 \\
\hline 8/20/08 & 7.46 & 15.5 & 8.92 & 46.22 & 160 \\
\hline 8/21/08 & 7.49 & 13.7 & 9.36 & 44.29 & 194 \\
\hline 8/22/08 & 7.45 & 12.1 & 9.79 & 45.20 & 163 \\
\hline 8/23/08 & 7.45 & 13.0 & 9.65 & 47.37 & 141 \\
\hline $8 / 24 / 08$ & 7.48 & 14.0 & 9.31 & 48.89 & 130 \\
\hline 8/25/08 & 7.51 & 13.8 & 9.32 & 49.03 & 135 \\
\hline 8/26/08 & 7.51 & 12.8 & 9.62 & 49.12 & 129 \\
\hline 8/27/08 & 7.51 & 13.5 & 9.43 & 49.52 & 122 \\
\hline 8/28/08 & 7.48 & 13.5 & 9.51 & 49.98 & 119 \\
\hline 8/29/08 & 7.47 & 14.7 & 9.16 & 50.16 & 121 \\
\hline 8/30/08 & 7.55 & 13.7 & 9.26 & 50.01 & 120 \\
\hline 8/31/08 & 7.56 & 11.6 & 9.73 & 49.94 & 115 \\
\hline 9/1/08 & 7.53 & 11.0 & 9.98 & 50.42 & 110 \\
\hline $9 / 2 / 08$ & 7.51 & 11.4 & 9.92 & 51.26 & 105 \\
\hline 9/3/08 & 7.47 & 11.8 & 9.76 & 51.96 & 101 \\
\hline 9/4/08 & 7.35 & 12.8 & 9.60 & 52.58 & 97 \\
\hline 9/5/08 & 7.41 & 13.1 & 9.46 & 53.06 & 94 \\
\hline 9/6/08 & 7.39 & 13.3 & 9.42 & 53.43 & 92 \\
\hline $9 / 7 / 08$ & 7.38 & 13.7 & 9.35 & 53.62 & 91 \\
\hline 9/8/08 & 7.41 & 13.1 & 9.43 & 53.90 & 89 \\
\hline 9/9/08 & 7.42 & 13.2 & 9.32 & 54.15 & 87 \\
\hline 9/10/08 & 7.41 & 12.4 & 9.53 & 54.34 & 84 \\
\hline 9/11/08 & 7.40 & 12.4 & 9.59 & 54.45 & 83 \\
\hline 9/12/08 & 7.43 & 12.8 & 9.42 & 54.88 & 81 \\
\hline 9/13/08 & 7.43 & 12.9 & 9.46 & 55.11 & 81 \\
\hline 9/14/08 & 7.43 & 12.3 & 9.63 & 55.14 & 80 \\
\hline 9/15/08 & 7.41 & 12.2 & 9.62 & 55.39 & 77 \\
\hline 9/16/08 & 7.41 & 12.4 & 9.58 & 55.60 & 76 \\
\hline 9/17/08 & 7.38 & 12.9 & 9.48 & 55.80 & 75 \\
\hline 9/18/08 & 7.43 & 13.1 & 9.38 & 55.91 & 74 \\
\hline 9/19/08 & 7.43 & 13.2 & 9.34 & 56.01 & 74 \\
\hline $9 / 20 / 08$ & 7.43 & 12.9 & 9.28 & 56.07 & 73 \\
\hline 9/21/08 & 7.47 & 12.5 & 9.45 & 55.48 & 77 \\
\hline $9 / 22 / 08$ & 7.46 & 11.3 & 9.68 & 55.58 & 75 \\
\hline $9 / 23 / 08$ & 7.43 & 9.3 & 10.25 & 55.92 & 74 \\
\hline $9 / 24 / 08$ & 7.46 & 9.5 & 10.15 & 56.22 & 73 \\
\hline
\end{tabular}




\begin{tabular}{|c|c|c|c|c|c|}
\hline 9/25/08 & 7.45 & 10.0 & 10.02 & 56.45 & 72 \\
\hline $9 / 26 / 08$ & 7.46 & 9.7 & 10.13 & 56.45 & 73 \\
\hline 9/27/08 & 7.44 & 10.0 & 10.06 & 56.83 & 71 \\
\hline $9 / 28 / 08$ & 7.43 & 9.7 & 10.06 & 57.39 & 69 \\
\hline 9/29/08 & 7.43 & 9.8 & 10.06 & 57.77 & 67 \\
\hline 9/30/08 & 7.45 & 9.7 & 10.06 & 58.02 & 66 \\
\hline 10/1/08 & 7.42 & 10.2 & 9.95 & 58.26 & 65 \\
\hline $10 / 2 / 08$ & 7.42 & 10.7 & 9.67 & 58.43 & 66 \\
\hline $10 / 3 / 08$ & 7.42 & 10.1 & 9.77 & 58.00 & 70 \\
\hline $10 / 4 / 08$ & 7.46 & 10.7 & 9.73 & 53.40 & 121 \\
\hline 10/5/08 & 7.46 & 9.4 & 10.20 & 50.33 & 125 \\
\hline $10 / 6 / 08$ & 7.45 & 8.5 & 10.42 & 52.15 & 98 \\
\hline $10 / 7 / 08$ & 7.41 & 8.6 & 10.47 & 52.09 & 98 \\
\hline $10 / 8 / 08$ & 7.28 & 6.8 & 10.99 & 51.45 & --- \\
\hline 10/9/08 & 7.30 & 6.2 & 11.06 & 53.14 & 96 \\
\hline 10/10/08 & 7.30 & 5.8 & 11.21 & 53.74 & 91 \\
\hline 10/11/08 & 7.29 & 5.0 & 11.42 & 54.45 & 87 \\
\hline 10/12/08 & 7.29 & 5.9 & 11.25 & 54.86 & 85 \\
\hline 10/13/08 & 7.29 & 6.7 & 11.10 & 54.87 & 85 \\
\hline 10/14/08 & 7.31 & 7.2 & 10.90 & 54.27 & 91 \\
\hline 10/15/08 & 7.29 & 5.8 & 11.22 & 54.30 & 87 \\
\hline 10/16/08 & 7.30 & 6.5 & 11.12 & 54.35 & 86 \\
\hline 10/17/08 & 7.29 & 8.0 & 10.72 & 54.44 & 86 \\
\hline 10/18/08 & 7.30 & 8.4 & 10.47 & 54.68 & 85 \\
\hline 10/19/08 & 7.30 & 6.3 & 11.03 & 55.04 & 84 \\
\hline $10 / 20 / 08$ & 7.30 & 6.4 & 10.97 & 54.96 & 85 \\
\hline 10/21/08 & 7.29 & 5.5 & 11.32 & 53.51 & 91 \\
\hline 10/22/08 & 7.31 & 5.1 & 11.50 & 54.97 & 84 \\
\hline 10/23/08 & 7.32 & 4.7 & 11.49 & 55.59 & 84 \\
\hline $10 / 24 / 08$ & 7.32 & 4.6 & 11.55 & 55.63 & 83 \\
\hline $10 / 25 / 08$ & 7.32 & 5.2 & 11.35 & 55.62 & 83 \\
\hline 10/26/08 & 7.31 & 4.4 & 11.63 & 55.95 & 81 \\
\hline 10/27/08 & 7.30 & 3.9 & 11.70 & 56.42 & 79 \\
\hline $10 / 28 / 08$ & 7.31 & 3.8 & 11.73 & 56.62 & 78 \\
\hline $10 / 29 / 08$ & 7.31 & 3.9 & 11.60 & 56.64 & 78 \\
\hline 10/30/08 & 7.31 & 4.3 & 11.48 & 56.81 & 77 \\
\hline 10/31/08 & 7.29 & 5.7 & 11.03 & 56.71 & 78 \\
\hline $11 / 1 / 08$ & 7.29 & 6.2 & 10.89 & 56.19 & 83 \\
\hline $11 / 2 / 08$ & 7.32 & 6.5 & 10.78 & 55.31 & 91 \\
\hline $11 / 3 / 08$ & 7.31 & 5.9 & 10.92 & 54.80 & 93 \\
\hline $11 / 4 / 08$ & 7.32 & 5.8 & 10.87 & 54.05 & 99 \\
\hline $11 / 5 / 08$ & 7.32 & 3.8 & 11.55 & 55.09 & 85 \\
\hline $11 / 6 / 08$ & 7.31 & 3.2 & 11.81 & 55.18 & 88 \\
\hline $11 / 7 / 08$ & 7.29 & 2.8 & 12.17 & 50.89 & 175 \\
\hline $11 / 8 / 08$ & 7.21 & 3.4 & 11.91 & 42.85 & 235 \\
\hline $11 / 9 / 08$ & 7.21 & 4.3 & 11.56 & 43.26 & 202 \\
\hline $11 / 10 / 08$ & 7.24 & 4.2 & 11.58 & 44.78 & 184 \\
\hline 11/11/08 & 7.25 & 4.3 & 11.58 & 45.71 & 175 \\
\hline 11/12/08 & 7.20 & 4.7 & 11.60 & 37.48 & 567 \\
\hline 11/13/08 & 7.26 & 3.9 & 11.94 & 35.33 & 573 \\
\hline $11 / 14 / 08$ & 7.26 & 2.8 & 12.41 & 37.98 & 441 \\
\hline
\end{tabular}




$\begin{array}{cccccc}11 / 15 / 08 & 7.28 & 3.2 & 12.20 & 39.29 & 394 \\ 11 / 16 / 08 & 7.30 & 3.3 & 12.09 & 39.70 & 365 \\ 11 / 17 / 08 & 7.32 & 3.3 & 12.10 & 40.28 & 345 \\ 11 / 18 / 08 & 7.34 & 3.4 & 12.04 & 40.71 & 328 \\ 11 / 19 / 08 & 7.34 & 2.7 & 12.29 & 41.45 & 304 \\ 11 / 20 / 08 & 7.36 & 3.0 & 12.10 & 42.08 & 290 \\ 11 / 21 / 08 & 7.37 & 2.8 & 12.19 & 42.48 & 275 \\ 11 / 22 / 08 & 7.37 & 2.7 & 12.18 & 43.12 & 262 \\ 11 / 23 / 08 & 7.39 & 1.5 & 12.52 & 44.43 & 228 \\ 11 / 24 / 08 & 7.40 & 0.7 & 12.75 & 45.43 & 218 \\ 11 / 25 / 08 & 7.39 & 1.0 & 12.68 & 45.55 & 218 \\ 11 / 26 / 08 & 7.40 & 0.7 & 12.72 & 46.08 & 200 \\ 11 / 27 / 08 & 7.40 & 0.2 & 12.82 & 47.40 & 182 \\ 11 / 28 / 08 & 7.40 & 0.8 & 12.80 & 47.15 & 186 \\ 11 / 29 / 08 & 7.40 & 1.6 & 12.59 & 47.14 & 185 \\ 11 / 30 / 08 & 7.41 & 2.3 & 12.40 & 46.63 & 186 \\ 12 / 1 / 08 & 7.42 & 2.5 & 12.19 & 46.54 & 183 \\ 12 / 2 / 08 & 7.42 & 2.7 & 12.14 & 46.60 & 176 \\ 12 / 3 / 08 & --- & --- & --- & -- & \end{array}$


Appendix D. Daily medians $(\mathrm{pH})$ or means of water quality parameters at the North Fork gage. Discharge is from the North Fork gage.

\begin{tabular}{|c|c|c|c|c|c|}
\hline Date & $\mathrm{pH}$ & Temperature (C) & $\begin{array}{c}\text { Dissolved } \\
\text { Oxygen } \\
(\mathrm{mg} / \mathrm{L})\end{array}$ & $\begin{array}{c}\text { Specific } \\
\text { Conductivity } \\
(\mathrm{mS} / \mathrm{cm})\end{array}$ & Discharge (cfs) \\
\hline 3/17/08 & & & & & 44 \\
\hline 3/18/08 & 7.38 & 2.0 & 11.96 & 47.60 & 44 \\
\hline 3/19/08 & 7.40 & 1.5 & 12.06 & 47.68 & 43 \\
\hline $3 / 20 / 08$ & 7.41 & 1.7 & 12.06 & 47.70 & 43 \\
\hline $3 / 21 / 08$ & 7.42 & 1.7 & 12.25 & 47.84 & 43 \\
\hline $3 / 22 / 08$ & 7.41 & 1.2 & 12.44 & 47.91 & 42 \\
\hline $3 / 23 / 08$ & 7.42 & 0.9 & 12.16 & 47.77 & 43 \\
\hline $3 / 24 / 08$ & 7.43 & 0.9 & 12.22 & 47.90 & 43 \\
\hline $3 / 25 / 08$ & 7.42 & 0.8 & 12.20 & 48.39 & 42 \\
\hline $3 / 26 / 08$ & 7.42 & 1.6 & 11.96 & 47.96 & 42 \\
\hline $3 / 27 / 08$ & 7.43 & 1.3 & 12.16 & 48.31 & 41 \\
\hline $3 / 28 / 08$ & 7.43 & -0.2 & 12.14 & 48.49 & 41 \\
\hline 3/29/08 & 7.43 & 0.5 & 12.13 & 48.38 & 41 \\
\hline 3/30/08 & 7.44 & 0.5 & 12.21 & 48.64 & 40 \\
\hline 3/31/08 & 7.43 & 0.4 & 12.35 & 48.67 & 40 \\
\hline 4/1/08 & 7.43 & 0.6 & 12.32 & 48.82 & 39 \\
\hline 4/2/08 & 7.44 & 1.3 & 12.17 & 48.43 & 39 \\
\hline 4/3/08 & 7.44 & 1.7 & 12.12 & 48.43 & 39 \\
\hline 4/4/08 & 7.44 & 2.0 & 11.92 & 48.41 & 39 \\
\hline 4/5/08 & 7.44 & 2.1 & 11.85 & 48.54 & 39 \\
\hline 4/6/08 & 7.44 & 2.8 & 11.76 & 48.24 & 39 \\
\hline 4/7/08 & 7.45 & 2.1 & 11.96 & 48.53 & 39 \\
\hline 4/8/08 & 7.45 & 1.9 & 11.97 & 48.59 & 38 \\
\hline 4/9/08 & 7.44 & 2.3 & 11.85 & 48.56 & 38 \\
\hline 4/10/08 & 7.42 & 2.6 & 11.99 & 48.39 & 39 \\
\hline 4/11/08 & 7.41 & 2.8 & 12.13 & 48.17 & 39 \\
\hline 4/12/08 & 7.41 & 3.4 & 12.01 & 47.67 & 43 \\
\hline 4/13/08 & 7.41 & 3.4 & 11.84 & 46.25 & 51 \\
\hline 4/14/08 & 7.37 & 3.1 & 11.71 & 44.90 & 63 \\
\hline 4/15/08 & 7.38 & 2.3 & 11.96 & 45.03 & 63 \\
\hline 4/16/08 & 7.37 & 2.7 & 12.05 & 44.96 & 64 \\
\hline 4/17/08 & 7.36 & 3.1 & 11.94 & 44.43 & 70 \\
\hline 4/18/08 & 7.36 & 2.4 & 11.90 & 44.11 & 76 \\
\hline 4/19/08 & 7.35 & 2.1 & 11.86 & 44.19 & 77 \\
\hline $4 / 20 / 08$ & 7.35 & 1.4 & 12.05 & 44.12 & 78 \\
\hline 4/21/08 & 7.35 & 1.3 & 12.11 & 44.31 & 77 \\
\hline $4 / 22 / 08$ & 7.35 & 1.7 & 12.04 & 44.45 & 76 \\
\hline 4/23/08 & 7.35 & 2.7 & 11.80 & 44.31 & 75 \\
\hline $4 / 24 / 08$ & 7.38 & 3.1 & 11.94 & 44.59 & 73 \\
\hline $4 / 25 / 08$ & 7.38 & 3.4 & 11.97 & 44.76 & 72 \\
\hline $4 / 26 / 08$ & 7.38 & 3.4 & 12.08 & 44.70 & 72 \\
\hline $4 / 27 / 08$ & 7.38 & 3.8 & 11.93 & 44.36 & 75 \\
\hline 4/28/08 & 7.37 & 3.8 & 11.74 & 43.97 & 82 \\
\hline
\end{tabular}




\begin{tabular}{|c|c|c|c|c|c|}
\hline 4/29/08 & 7.39 & 3.5 & 11.68 & 43.19 & 91 \\
\hline 4/30/08 & 7.38 & 3.1 & 11.85 & 43.28 & 92 \\
\hline $5 / 1 / 08$ & 7.37 & 3.2 & 11.97 & 43.04 & 99 \\
\hline $5 / 2 / 08$ & 7.37 & 3.3 & 11.99 & 42.68 & 113 \\
\hline $5 / 3 / 08$ & 7.38 & 3.4 & 11.93 & 42.54 & 127 \\
\hline $5 / 4 / 08$ & 7.36 & 4.1 & 11.83 & 41.88 & 158 \\
\hline $5 / 5 / 08$ & 7.34 & 3.6 & 11.85 & 40.01 & 227 \\
\hline $5 / 6 / 08$ & 7.25 & 3.4 & 11.86 & 38.47 & 304 \\
\hline $5 / 7 / 08$ & 7.16 & 3.4 & 11.88 & 37.17 & 382 \\
\hline $5 / 8 / 08$ & 7.16 & 3.3 & 11.91 & 36.98 & 410 \\
\hline $5 / 9 / 08$ & 7.17 & 3.5 & 11.92 & 37.31 & 397 \\
\hline $5 / 10 / 08$ & 7.16 & 3.6 & 11.85 & 37.51 & 393 \\
\hline $5 / 11 / 08$ & 7.20 & 3.9 & 11.81 & 37.45 & 393 \\
\hline $5 / 12 / 08$ & 7.19 & 4.0 & 11.85 & 37.54 & 386 \\
\hline 5/13/08 & 7.14 & 3.9 & 11.54 & --- & 410 \\
\hline $5 / 14 / 08$ & 7.07 & 4.2 & 11.58 & --- & 491 \\
\hline $5 / 15 / 08$ & 7.02 & 3.6 & 11.57 & --- & 738 \\
\hline 5/16/08 & 6.97 & 3.5 & 11.59 & --- & 1109 \\
\hline $5 / 17 / 08$ & 6.90 & 3.4 & 11.57 & --- & 1629 \\
\hline 5/18/08 & 6.83 & 3.6 & 11.54 & --- & 2318 \\
\hline 5/19/08 & 6.77 & 3.9 & 11.40 & --- & 2133 \\
\hline $5 / 20 / 08$ & 6.80 & 3.9 & 11.27 & 31.51 & 2016 \\
\hline $5 / 21 / 08$ & 6.89 & 4.0 & 11.26 & 32.53 & 1630 \\
\hline $5 / 22 / 08$ & 6.94 & 4.1 & 11.10 & 33.91 & 1298 \\
\hline $5 / 23 / 08$ & 6.91 & 4.0 & 11.08 & 34.29 & 1147 \\
\hline $5 / 24 / 08$ & 6.94 & 4.4 & 11.09 & 33.60 & 1121 \\
\hline $5 / 25 / 08$ & 6.97 & 4.5 & 11.18 & 32.21 & 1193 \\
\hline $5 / 26 / 08$ & 7.00 & 4.5 & 11.18 & 31.73 & 1291 \\
\hline $5 / 27 / 08$ & 7.00 & 4.7 & 11.48 & --- & 1356 \\
\hline $5 / 28 / 08$ & 7.02 & 4.5 & 11.78 & --- & 1397 \\
\hline 5/29/08 & 7.03 & 4.9 & 11.69 & --- & 1389 \\
\hline 5/30/08 & 7.04 & 4.7 & 11.79 & --- & 1386 \\
\hline 5/31/08 & 7.02 & 4.8 & 11.76 & --- & 1291 \\
\hline 6/1/08 & 7.02 & 5.1 & 11.68 & --- & 1253 \\
\hline $6 / 2 / 08$ & 7.05 & 4.6 & 11.77 & --- & 1225 \\
\hline $6 / 3 / 08$ & 7.12 & 4.3 & 11.77 & --- & 1106 \\
\hline $6 / 4 / 08$ & 7.13 & 4.6 & 11.72 & --- & 922 \\
\hline $6 / 5 / 08$ & 7.17 & 4.5 & 11.70 & --- & 797 \\
\hline 6/6/08 & 7.18 & 4.5 & 11.67 & --- & 730 \\
\hline $6 / 7 / 08$ & 7.17 & 5.0 & 11.61 & --- & 670 \\
\hline 6/8/08 & 7.17 & 5.1 & 11.61 & --- & 635 \\
\hline 6/9/08 & 7.18 & 4.7 & 11.60 & --- & 623 \\
\hline 6/10/08 & 7.13 & 4.7 & 11.72 & --- & 605 \\
\hline 6/11/08 & 7.11 & 5.5 & 11.53 & 35.54 & 588 \\
\hline 6/12/08 & 7.11 & 6.0 & 11.42 & 35.07 & 603 \\
\hline 6/13/08 & 7.10 & 6.4 & 11.29 & 33.46 & 674 \\
\hline 6/14/08 & 7.08 & 5.4 & 11.58 & 32.07 & 757 \\
\hline 6/15/08 & 7.08 & 5.7 & 11.46 & 31.97 & 768 \\
\hline 6/16/08 & 7.07 & 5.7 & 11.45 & 31.70 & 786 \\
\hline 6/17/08 & 7.09 & 5.7 & 11.50 & 31.65 & 787 \\
\hline 6/18/08 & 7.10 & 5.3 & 11.62 & 32.01 & 766 \\
\hline 6/19/08 & 7.10 & 5.3 & 11.62 & 32.66 & 718 \\
\hline
\end{tabular}




\begin{tabular}{|c|c|c|c|c|c|}
\hline 6/20/08 & 7.10 & 6.2 & 11.37 & 33.05 & 684 \\
\hline 6/21/08 & 7.10 & 5.7 & 11.46 & 32.40 & 716 \\
\hline 6/22/08 & 7.11 & 6.3 & 11.33 & 31.79 & 750 \\
\hline $6 / 23 / 08$ & 7.13 & 5.4 & 11.56 & 31.65 & 760 \\
\hline 6/24/08 & 7.13 & 6.2 & 11.35 & 32.36 & 710 \\
\hline 6/25/08 & 7.12 & 6.1 & 11.33 & 32.45 & 622 \\
\hline 6/26/08 & 7.18 & 6.6 & 11.20 & 32.26 & 577 \\
\hline $6 / 27 / 08$ & 7.18 & 7.4 & 11.07 & 31.56 & 618 \\
\hline 6/28/08 & 7.13 & 7.7 & 11.00 & 29.96 & 717 \\
\hline 6/29/08 & 7.11 & 7.8 & 10.96 & 28.90 & 805 \\
\hline 6/30/08 & 7.10 & 8.0 & 10.90 & 28.27 & 892 \\
\hline $7 / 1 / 08$ & 7.12 & 8.0 & 10.89 & 28.36 & 950 \\
\hline $7 / 2 / 08$ & 7.11 & 8.1 & 10.87 & 28.74 & 895 \\
\hline $7 / 3 / 08$ & 7.15 & 8.2 & 10.78 & 28.87 & 838 \\
\hline $7 / 4 / 08$ & 7.19 & 7.9 & 10.85 & 29.39 & 772 \\
\hline $7 / 5 / 08$ & 7.23 & 7.2 & 11.04 & 30.88 & 653 \\
\hline 7/6/08 & 7.25 & 7.9 & 10.87 & 32.05 & 568 \\
\hline $7 / 7 / 08$ & 7.25 & 8.0 & 10.87 & 32.66 & 516 \\
\hline 7/8/08 & 7.20 & 8.5 & 10.78 & 32.92 & 514 \\
\hline $7 / 9 / 08$ & 7.17 & 9.0 & 10.58 & 32.60 & 538 \\
\hline 7/10/08 & 7.19 & 8.4 & 10.70 & 32.46 & 527 \\
\hline $7 / 11 / 08$ & 7.22 & 7.3 & 11.06 & 33.75 & 464 \\
\hline $7 / 12 / 08$ & 7.22 & 8.5 & 10.76 & 34.66 & 412 \\
\hline $7 / 13 / 08$ & 7.20 & 9.2 & 10.53 & 34.75 & 397 \\
\hline $7 / 14 / 08$ & 7.22 & 9.1 & 10.52 & 34.41 & 395 \\
\hline $7 / 15 / 08$ & 7.19 & 9.1 & 10.55 & 34.51 & 372 \\
\hline $7 / 16 / 08$ & 7.17 & 9.3 & 10.57 & 34.66 & 351 \\
\hline $7 / 17 / 08$ & 7.23 & 9.2 & 10.57 & 34.92 & 334 \\
\hline 7/18/08 & 7.23 & 9.1 & 10.53 & 35.45 & 310 \\
\hline 7/19/08 & 7.24 & 9.3 & 10.48 & 35.95 & 289 \\
\hline $7 / 20 / 08$ & 7.24 & 9.9 & 10.32 & 36.17 & 280 \\
\hline $7 / 21 / 08$ & 7.24 & 10.1 & 10.25 & 36.12 & 276 \\
\hline $7 / 22 / 08$ & 7.26 & 10.5 & 10.16 & 35.85 & 272 \\
\hline $7 / 23 / 08$ & 7.27 & 10.0 & 10.33 & 35.77 & 264 \\
\hline $7 / 24 / 08$ & 7.26 & 9.8 & 10.41 & 36.52 & 245 \\
\hline $7 / 25 / 08$ & 7.26 & 10.0 & 10.34 & 37.18 & 232 \\
\hline $7 / 26 / 08$ & 7.27 & 10.3 & 10.23 & 37.20 & 229 \\
\hline $7 / 27 / 08$ & 7.26 & 10.5 & 10.18 & 37.08 & 226 \\
\hline $7 / 28 / 08$ & 7.27 & 9.8 & 10.35 & 37.30 & 216 \\
\hline $7 / 29 / 08$ & 7.38 & 9.1 & 10.48 & 37.85 & 201 \\
\hline 7/30/08 & 7.43 & 9.3 & 10.45 & 38.30 & 188 \\
\hline 7/31/08 & 7.44 & 8.9 & 10.52 & 38.97 & 168 \\
\hline 8/1/08 & 7.45 & 10.0 & 10.25 & 39.47 & 164 \\
\hline $8 / 2 / 08$ & 7.43 & 8.8 & 10.56 & 39.28 & 157 \\
\hline 8/3/08 & 7.42 & 9.4 & 10.45 & 40.11 & 138 \\
\hline $8 / 4 / 08$ & 7.43 & 9.9 & 10.35 & 40.98 & 124 \\
\hline 8/5/08 & 7.38 & 10.5 & 10.14 & 41.19 & 120 \\
\hline 8/6/08 & 7.36 & 10.8 & 10.03 & 40.55 & 124 \\
\hline $8 / 7 / 08$ & 7.40 & 11.2 & 9.94 & 39.82 & 134 \\
\hline 8/8/08 & 7.41 & 11.3 & 9.87 & 39.06 & 146 \\
\hline 8/9/08 & 7.45 & 11.0 & 9.99 & 38.33 & 157 \\
\hline 8/10/08 & 7.48 & 9.9 & 10.29 & 39.11 & 130 \\
\hline
\end{tabular}




\begin{tabular}{|c|c|c|c|c|c|}
\hline 8/11/08 & 7.47 & 9.2 & 10.43 & 40.54 & 107 \\
\hline 8/12/08 & 7.48 & 10.0 & 10.24 & 41.48 & 96 \\
\hline 8/13/08 & 7.47 & 11.0 & 10.00 & 41.65 & 95 \\
\hline 8/14/08 & 7.45 & 11.9 & 9.84 & 41.42 & 96 \\
\hline 8/15/08 & 7.42 & 12.2 & 9.76 & 40.62 & 106 \\
\hline 8/16/08 & 7.40 & 12.7 & 9.61 & 39.51 & 118 \\
\hline 8/17/08 & 7.39 & 12.6 & 9.61 & 38.66 & 126 \\
\hline 8/18/08 & 7.43 & 12.0 & 9.72 & 38.51 & 125 \\
\hline 8/19/08 & 7.46 & 11.5 & 9.86 & 38.74 & 118 \\
\hline 8/20/08 & 7.47 & 10.8 & 9.99 & 38.66 & 152 \\
\hline 8/21/08 & 7.46 & 9.2 & 10.38 & 37.54 & 172 \\
\hline 8/22/08 & 7.51 & 8.0 & 10.75 & 39.79 & 112 \\
\hline 8/23/08 & 7.51 & 9.3 & 10.43 & 41.74 & 89 \\
\hline 8/24/08 & 7.53 & 9.9 & 10.23 & 42.58 & 86 \\
\hline 8/25/08 & 7.54 & 10.0 & 10.20 & 42.06 & 89 \\
\hline 8/26/08 & 7.55 & 8.7 & 10.54 & 41.92 & 84 \\
\hline 8/27/08 & 7.57 & 8.9 & 10.47 & 42.55 & 82 \\
\hline 8/28/08 & 7.52 & 9.3 & 10.44 & 43.02 & 79 \\
\hline 8/29/08 & 7.53 & 10.4 & 10.10 & 42.61 & 83 \\
\hline 8/30/08 & 7.56 & 9.3 & 10.33 & 41.90 & 82 \\
\hline 8/31/08 & 7.57 & 7.1 & 10.86 & 42.59 & 76 \\
\hline 9/1/08 & 7.57 & 7.1 & 10.94 & 43.67 & 71 \\
\hline $9 / 2 / 08$ & 7.57 & 7.3 & 10.92 & 44.48 & 67 \\
\hline $9 / 3 / 08$ & 7.53 & 8.0 & 10.73 & 45.17 & 65 \\
\hline $9 / 4 / 08$ & 7.33 & 8.8 & 10.55 & 45.53 & 63 \\
\hline 9/5/08 & 7.36 & 9.2 & 10.45 & 45.72 & 61 \\
\hline 9/6/08 & 7.35 & 9.5 & 10.39 & 45.69 & 61 \\
\hline 9/7/08 & 7.34 & 9.8 & 10.30 & 45.75 & 60 \\
\hline 9/8/08 & 7.37 & 8.9 & 10.48 & 45.93 & 59 \\
\hline 9/9/08 & 7.36 & 9.1 & 10.34 & 46.16 & 58 \\
\hline 9/10/08 & 7.38 & 8.1 & 10.66 & 46.40 & 57 \\
\hline 9/11/08 & 7.36 & 8.3 & 10.62 & 46.60 & 54 \\
\hline 9/12/08 & 7.37 & 8.8 & 10.45 & 46.85 & 51 \\
\hline 9/13/08 & 7.38 & 8.6 & 10.58 & 46.66 & 51 \\
\hline $9 / 14 / 08$ & 7.38 & 8.3 & 10.67 & 46.66 & 50 \\
\hline 9/15/08 & 7.38 & 8.2 & 10.68 & 47.06 & 50 \\
\hline 9/16/08 & 7.38 & 8.4 & 10.62 & 47.12 & 49 \\
\hline 9/17/08 & 7.38 & 8.9 & 10.48 & 47.12 & 49 \\
\hline 9/18/08 & 7.38 & 9.3 & 10.36 & 46.95 & 49 \\
\hline 9/19/08 & 7.38 & 9.3 & 10.34 & 46.63 & 49 \\
\hline 9/20/08 & 7.40 & 9.5 & 10.25 & 46.60 & 49 \\
\hline 9/21/08 & 7.42 & 9.2 & 10.35 & 46.22 & 50 \\
\hline 9/22/08 & 7.42 & 7.5 & 10.79 & 46.60 & 49 \\
\hline 9/23/08 & 7.42 & 5.5 & 11.32 & 47.38 & 47 \\
\hline 9/24/08 & 7.41 & 6.2 & 11.10 & 47.93 & 46 \\
\hline 9/25/08 & 7.41 & 7.4 & 10.79 & 48.16 & 47 \\
\hline 9/26/08 & 7.42 & 6.6 & 11.07 & 48.42 & 46 \\
\hline 9/27/08 & 7.41 & 6.4 & 11.10 & 48.61 & 45 \\
\hline 9/28/08 & 7.41 & 5.8 & 11.30 & 49.14 & 44 \\
\hline 9/29/08 & 7.42 & 5.9 & 11.26 & 49.39 & 43 \\
\hline 9/30/08 & 7.41 & 6.2 & 11.18 & 49.52 & 43 \\
\hline 10/1/08 & 7.40 & 7.2 & 10.89 & 49.53 & --- \\
\hline
\end{tabular}




\begin{tabular}{|c|c|c|c|c|c|}
\hline 10/2/08 & 7.41 & 7.7 & 10.64 & 49.38 & --- \\
\hline 10/3/08 & 7.39 & 7.8 & 10.57 & 48.29 & --- \\
\hline 10/4/08 & 7.35 & 7.9 & 10.52 & 43.71 & --- \\
\hline 10/5/08 & 7.39 & 6.6 & 10.95 & 42.73 & --- \\
\hline 10/6/08 & 7.42 & 5.5 & 11.30 & 45.05 & --- \\
\hline 10/7/08 & 7.41 & 5.6 & 11.30 & 44.03 & -- \\
\hline 10/8/08 & 7.42 & 4.1 & 11.73 & 45.07 & --- \\
\hline 10/9/08 & 7.43 & 3.4 & 11.85 & 46.23 & --- \\
\hline 10/10/08 & 7.43 & 2.9 & 12.04 & 46.74 & --- \\
\hline 10/11/08 & 7.43 & 2.1 & 12.25 & 47.46 & --- \\
\hline $10 / 12 / 08$ & 7.42 & 3.2 & 11.96 & 47.57 & --- \\
\hline $10 / 13 / 08$ & 7.42 & 4.5 & 11.67 & 47.39 & --- \\
\hline 10/14/08 & 7.44 & 4.9 & 11.55 & 46.41 & -- \\
\hline 10/15/08 & 7.42 & 3.2 & 11.95 & 46.68 & -- \\
\hline 10/16/08 & 7.42 & 4.1 & 11.83 & 46.89 & --- \\
\hline 10/17/08 & 7.41 & 5.5 & 11.32 & 47.00 & --- \\
\hline 10/18/08 & 7.44 & 5.6 & 11.19 & 47.21 & --- \\
\hline 10/19/08 & 7.44 & 3.2 & 11.97 & 47.33 & --- \\
\hline 10/20/08 & 7.44 & 3.5 & 11.77 & 47.00 & --- \\
\hline 10/21/08 & 7.42 & 2.4 & 12.16 & 46.99 & -- \\
\hline 10/22/08 & 7.44 & 2.1 & 12.00 & 48.05 & -- \\
\hline $10 / 23 / 08$ & 7.45 & 2.2 & 11.86 & 48.16 & --- \\
\hline $10 / 24 / 08$ & 7.45 & 2.5 & 11.84 & 48.00 & --- \\
\hline $10 / 25 / 08$ & 7.45 & 3.4 & 11.61 & 48.03 & --- \\
\hline 10/26/08 & 7.46 & 2.0 & 12.11 & 48.52 & --- \\
\hline 10/27/08 & 7.45 & 1.5 & 12.16 & 48.87 & -- \\
\hline 10/28/08 & 7.45 & 1.8 & 12.06 & 48.90 & --- \\
\hline $10 / 29 / 08$ & 7.45 & 2.2 & 11.87 & 48.86 & --- \\
\hline 10/30/08 & 7.44 & 2.9 & 11.68 & 48.87 & --- \\
\hline $10 / 31 / 08$ & 7.43 & 4.3 & 11.28 & 48.88 & --- \\
\hline 11/1/08 & 7.43 & 4.1 & 11.26 & 48.73 & --- \\
\hline 11/2/08 & 7.46 & 4.6 & 11.08 & 47.74 & -- \\
\hline 11/3/08 & 7.47 & 3.8 & 11.29 & 47.14 & --- \\
\hline 11/4/08 & 7.46 & 3.1 & 11.40 & 46.82 & --- \\
\hline 11/5/08 & 7.47 & 1.2 & 12.10 & 48.21 & --- \\
\hline 11/6/08 & 7.44 & 0.3 & 12.38 & 47.30 & --- \\
\hline 11/7/08 & 7.37 & 1.1 & 12.25 & 41.38 & --- \\
\hline 11/8/08 & 7.22 & 2.4 & 11.75 & 36.92 & --- \\
\hline 11/9/08 & 7.27 & 2.9 & 11.59 & 38.45 & --- \\
\hline 11/10/08 & 7.32 & 3.1 & 11.60 & 39.53 & --- \\
\hline 11/11/08 & 7.31 & 2.5 & 11.75 & 40.10 & --- \\
\hline $11 / 12 / 08$ & 7.11 & 3.0 & 11.63 & 33.66 & --- \\
\hline $11 / 13 / 08$ & 7.24 & 2.6 & 11.89 & 34.48 & --- \\
\hline 11/14/08 & 7.26 & 2.0 & 12.20 & 36.09 & --- \\
\hline 11/15/08 & 7.29 & 2.8 & 11.90 & 36.86 & -- \\
\hline 11/16/08 & 7.33 & 2.8 & 11.85 & 37.31 & -- \\
\hline 11/17/08 & 7.36 & 2.5 & 11.90 & 37.81 & --- \\
\hline 11/18/08 & 7.38 & 2.8 & 11.81 & 38.21 & --- \\
\hline 11/19/08 & 7.38 & 1.8 & 12.18 & 38.92 & --- \\
\hline $11 / 20 / 08$ & 7.38 & 2.3 & 11.90 & 39.32 & --- \\
\hline 11/21/08 & 7.39 & 1.6 & 12.13 & 39.80 & -- \\
\hline $11 / 22 / 08$ & 7.41 & 1.6 & 12.10 & 40.23 & --- \\
\hline
\end{tabular}




$\begin{array}{cccccc}11 / 23 / 08 & 7.41 & 0.3 & 12.52 & 41.54 & --- \\ 11 / 24 / 08 & 7.41 & 0.0 & 12.58 & 42.05 & --- \\ 11 / 25 / 08 & 7.42 & 0.4 & 12.42 & 42.13 & -- \\ 11 / 26 / 08 & 7.41 & 0.0 & 12.59 & 42.81 & -- \\ 11 / 27 / 08 & 7.45 & -0.3 & 12.61 & 42.75 & --- \\ 11 / 28 / 08 & 7.43 & 0.2 & 12.55 & 43.38 & -- \\ 11 / 29 / 08 & 7.43 & 1.4 & 12.24 & 43.11 & -- \\ 11 / 30 / 08 & 7.42 & 1.7 & 12.13 & 42.40 & -- \\ 12 / 1 / 08 & 7.43 & 1.6 & 12.00 & 42.32 & -- \\ 12 / 2 / 08 & 7.41 & 2.2 & 11.92 & 42.48 & -- \\ 12 / 3 / 08 & --- & --- & -- & -- & --\end{array}$

